Japan. J. Math.

Vol. 23, No. 2, 1997

\title{
Non-smooth hyperbolic mixed problems of the second order
}

\author{
By Andrzej CHRZȨSZCZYK \\ (Received September 8, 1995) \\ (from Tsukuba Journal of Mathematics)
}

\section{Introduction}

It is well known that any mathematical model of arbitrary dynamical theory should satisfy the requirement of the existence of solutions to the corresponding initial and initial-boundary value problems. Unfortunately the known existence theorems are not strong enough to describe global solutions in elasticity or thermoelasticity, in the general, multidimensional and nonliner case. In some special cases, for example for special materials and very smooth and small initial data one can prove the global in time existence (cf. for example $[10,25,32]$ ) but generally, the global solvability in the class of smooth functions is not expected. Furthermore the experience with formation of singularities (cf. $[9,13,26,27]$ ) suggests that a sufficiently general, non-linear theory of evolution problems should admit the nonclassical solutions, i.e. the solutions with discontinuities in some derivatives occuring in the corresponding equations. Unfortunately almost all papers concerning the existence of solutions for nonlinear, multidimensional hyperbolic and hyperbolicparabolic problems of the second order are restricted to the classical solutions, $\mathrm{cf}$. for example $[1,3-4,6-8,10-12,14-15,17-18,22-23,25,28-32]$. An exceptional character is found in the papers $[16,21]$ but it seems that the results contained there do not cover the systems of elasticity or thermoelasticity.

The main purpose of this article is to present some methods of proving the local in time existence results for initial-boundary value problems for second order quasilinear hyperbolic systems modeled on the system of elasticity. The solutions we consider are relatively regular but they may possess singularities in derivatives of the second order and the coefficients of the investigated quasi-linear systems are not necessarily Lipschitz-continuous. As a consequence the methods developed in the papers quoted above are not aplicable and we are forced to apply a new technique. In the case of the initial problem (cf. [5]) it is possible to give relatively short proof of the existence of non-classical solutions for a class of systems generalizing the systems of elasticity and thermoelasticity, using the methods from the book [34].

The case of the mixed, initial-boundary value problem is more complicated 
and therefore in the present paper we restrict ourselves to the hyperbolic system of the type of elasticity. The proof of the existence of solutions to the non-linear mixed problem (cf. Section 4) is based on some quite delicate results concerning the corresponding linear problem. This is the reason for which the largest part of this article is devoted to the analysis of the linear, hyperbolic, mixed problems with nonsmooth coefficients (cf. Sections 2-3). Sections 5, 6 contain some technical material frequently used in the main text. A more precise description of the contents can be found in the table of contents.

\section{Table of contents}

1. Introduction

2. Regularization procedure for hyperbolic mixed problems

2.1. Introductory remarks

2.2. Basic assumptions

2.3. Formulation of the regularization theorem

2.4. Proof of the regularization theorem

3. Energy estimates for non-smooth Cauchy-Dirichlet problem

3.1. Introductory remarks

3.2. Basic assumptions

3.3. The basic estimate

3.4. First step of the proof

3.5. Application of elliptic estimates with $L^{2}$ dependence on $t$

4. Existence theorems for non-smooth Cauchy-Dirichlet problem

4.1. Introductory remarks

4.2. Existence theorem for the linear problem

4.3. Existence theorem for the quasi-linear problem

5. Function spaces and product estimates

5.1. Some properties of Sobolev spaces

5.2. Some properties of traces

5.3. Some product estimates

5.4. Some further product estimates

5.5. Convergence of commutators

6. Some elliptic estimates

6.1. Elliptic estimates independent of $t$

6.2. Elliptic estimates with $L^{2}$ dependence on $t$

References

\section{Regularization procedure for hyperbolic mixed problems}

\subsection{Introductory remarks}

In some methods of proving the existence of solutions to evolution problems 
it is necessary to approximate the initial values, the coefficients or the right hand side by smooth functions. When the mixed, initial-boundary value problems are considered, an essential trouble occurs. The usual approximation procedure leads to smooth data, which do not satisfy the boundary or compatibility conditions. To overcome this difficulty, some additional ideas are needed, cf. for example $[8,15$, 22-23].

In the present section we consider the linear, second order, mixed problems with homogeneous Dirichlet boundary conditions and nonhomogeneous Neumann conditions. Our main purpose is to present some smoothing procedure, which preserves the boundary and compatibility conditions and which is applicable in the case when the coefficients are less smooth than those in $[1,4,8,15,17-18,22-23$, 28-32]. In the next section we shall show that the presented procedure is in an accordance with some energy method.

\subsection{Basic assumptions}

In this section we shall consider the two basic mixed problems for linear, hyperbolic systems of the second order, the Neumann and Dirichlet problems:

$$
\begin{array}{lll}
u^{(2)}=a \partial^{2} u+f, & \text { in } \Omega \times I, & \\
b \partial u=g, & \text { on } \partial \Omega \times I, & \text { (Neumann b.c.) }, \\
u=0, & \text { on } \partial \Omega \times I, \quad \text { (Dirichlet b.c.) }, \\
u(0)=u^{0}, \quad u^{(1)}(0)=u^{1}, & \text { in } \Omega .
\end{array}
$$

Here $\Omega \subset R^{n}, n \geq 2$ is a bounded domain with $C^{\infty}$ and compact boundary $\partial \Omega$, $I=(0, T)$ denotes a fixed, finite interval, $u^{(k)}, k=0,1, \ldots$, denotes the $k$-th derivative with respect to $t \in I$. Furthermore

$$
\begin{aligned}
a \partial^{2} u & =\sum_{p, q=1}^{n+1} a_{p, q} \partial_{p} \partial_{q} u, \\
b \partial u & =\sum_{p, q=1}^{n} \nu_{p} a_{p, q} \partial_{q} u
\end{aligned}
$$

where $\partial_{p}=\partial / \partial_{x_{p}}$ denotes the partial derivative with respect to $x_{p}$, for $p=1, \ldots, n$, $\partial_{n+1} \varphi=\varphi$ and $\nu=\left(\nu_{1}, \ldots, \nu_{n}\right)$ denotes the unit outward normal to the boundary, which is considered as a $C_{0}^{\infty}\left(R^{n}\right)$ vector field. The unknown function $u=u(x, t)$ and the data $u^{i}=u^{i}(x), i=0,1, f=f(x, t), g=g\left(x^{\prime}, t\right), t \in I, x \in \Omega, x^{\prime} \in \partial \Omega$ take values in $R^{m}$. The symbols $a_{p, q}=a_{p, q}(x, t), p, q=1, \ldots, n+1, x \in \Omega, t \in I$ denote given, real, $m \times m$ matrices which are symmetric for $p, q \neq n+1$.

We shall assume that a real number $s$ and an integer $L$ are given which satisfy 
the relations

$$
\begin{gathered}
\qquad \frac{n}{2}<s \leq L-1, \\
r_{j}+\frac{3}{2} \text { are noninteger for } j=0, \ldots, L-1, \\
\text { where } r_{j}=\frac{s}{L}\left(L-j-\frac{1}{2}\right), \quad j=0, \ldots, L-1 .
\end{gathered}
$$

To formulate our regularity hypotheses let us introduce some additional notations. If $k=0,1, \ldots$ and $r \in R$ then one can define the anisotropic Sobolev spaces

$$
H^{r, k}(\Omega \times I)=\left\{u: u \in L^{2}\left(I, H^{r}(\Omega)\right), u^{(k)} \in L^{2}\left(I, H^{0}(\Omega)\right)\right\}
$$

with the norm

$$
\|u\|_{H^{r, k}(\Omega \times I)}^{2}=\int_{I}\left(\|u(t)\|_{H^{r}(\Omega)}^{2}+\left\|u^{(k)}(t)\right\|_{H^{0}(\Omega)}^{2}\right) d t .
$$

Here $H^{r}(\Omega)$ and $\|\cdot\|_{H^{r}(\Omega)}$ denote the usual Sobolev space and its norm respectively, as defined for example in [2], Chapt. 2 or [20], Chapt. 1, Sect. 9.1. The symbol $L^{\alpha}(I, X)$ denotes the space of all strongly measurable functions of $t$ with values in $X$, which are integrable with the power $\alpha$ for $1 \leq \alpha<\infty$ and are essentially bounded for $\alpha=\infty$.

We shall assume that the data occuring in (2.2.1) satisfy the following conditions

the boundary function $g$ is a trace on $\partial \Omega \times I$ of some function $\bar{g} \in H^{s+1, L}(\Omega \times I)$,

$$
\begin{gathered}
\partial a \in H^{s, L-1}(\Omega \times I), \quad a^{(L)} \in L^{2}\left(I, H^{0}(\Omega)\right), \\
a=\left\{a_{p, q}: p, q=1, \ldots, n+1\right\}, \quad \partial=\left(\partial_{1}, \ldots, \partial_{n}\right), \\
a^{(j)}(0) \in H^{r_{j}+1}(\Omega), \quad j=0, \ldots, L-1, \\
u^{j} \in H^{r_{j}+2}(\Omega), \quad j=0, \ldots, L-1, \\
u^{L} \in H^{1}(\Omega) \cap H^{r_{L-2}}(\Omega), \quad u^{L+1} \in H^{r_{L-1}}(\Omega) .
\end{gathered}
$$


Here $u^{2}, \ldots, u^{L+1}$ are defined with the use of the recursive formula

$$
u^{j+2}=\sum_{i=0}^{j}\left(\begin{array}{l}
j \\
i
\end{array}\right) a^{(i)}(0) \partial^{2} u^{j-i}+f^{(j)}(0), \quad j=0, \ldots, L-1 .
$$

Lemma 5.3.2 from Section 5.3, below shows that (2.2.10) is meaningful if (2.2.6)(2.2.9) are satisfied. Similarly, using Lemma 5.3.3 one can show that if (2.2.6)(2.2.9) are satisfied then the compatibility conditions are meaningful:

$$
\sum_{i=0}^{j}\left(\begin{array}{l}
j \\
i
\end{array}\right) b^{(i)}(0) \partial u^{j-i}=g^{(j)}(0), \quad \text { on } \partial \Omega, \quad j=0, \ldots, L-1
$$

$$
\text { for the Neumann b.c., }
$$

$$
u^{j}=0, \quad \text { on } \partial \Omega, \quad j=0, \ldots, L, \text { for the Dirichlet b.c. }
$$

It is clear that the regularity assumptions (2.2.6)-(2.2.9) and the compatibility conditions (2.2.10), (2.2.11) are not sufficient for solvability of (2.2.1). We shall assume also that the operator $a \partial^{2}$ is elliptic i.e. there exists $\delta>0$ such that

$$
\delta I_{m} \sum_{p=1}^{n}\left(\xi^{p}\right)^{2} \leq \sum_{p, q=1}^{n} a_{p, q}(x, t) \xi^{p} \xi^{q} \leq \delta^{-1} I_{m} \sum_{p=1}^{n}\left(\xi^{p}\right)^{2},
$$

for arbitrary $x \in \Omega, t \in I,\left(\xi_{1}, \ldots, \xi_{n}\right) \in R^{n}$. Here $I_{m}$ denotes the unit $m \times m$ matrix.

\subsection{Formulation of the regularization theorem}

Our main purpose is to construct a family of functions

$$
\begin{gathered}
f_{\alpha}, \bar{g}_{\alpha}, a_{\alpha}, u_{\alpha}^{j}, \quad j=0, \ldots, L+1, \\
a_{\alpha}=\left\{a_{p, q, \alpha}: p, q=1, \ldots, n+1\right\}, \quad \alpha \in(0,1),
\end{gathered}
$$

satisfying for $\alpha \in(0,1)$ the regularity conditions

$$
\begin{gathered}
f_{\alpha}, \bar{g}_{\alpha}, a_{\alpha} \in C^{\infty}(\bar{\Omega} \times \bar{I}), \\
u_{\alpha}^{j} \in C^{\infty}(\bar{\Omega}), \quad j=0, \ldots, L+1,
\end{gathered}
$$

and the compatibility conditions

$$
\sum_{i=0}^{j}\left(\begin{array}{l}
j \\
i
\end{array}\right) b_{\alpha}^{(i)}(0) \partial u_{\alpha}^{j-i}=g_{\alpha}^{(j)}(0), \quad \text { on } \partial \Omega, \quad j=0, \ldots, L-1
$$

for the Neumann b.c., $u_{\alpha}^{j}=0, \quad$ on $\partial \Omega, \quad j=0, \ldots, L, \quad$ for the Dirichlet b.c., 
where $g_{\alpha}$ denotes the trace of $\bar{g}_{\alpha}$ on $\partial \Omega \times I$ and

$$
\begin{gathered}
b_{\alpha} \partial u=\sum_{p, q=1}^{n} \nu_{p} a_{p, q, \alpha} \partial_{q} u \\
u_{\alpha}^{j+2}=\sum_{i=0}^{j}\left(\begin{array}{l}
j \\
i
\end{array}\right) a_{\alpha}^{(i)}(0) \partial^{2} u_{\alpha}^{j-i}+f_{\alpha}^{(j)}(0), \quad j=0, \ldots, L-1 .
\end{gathered}
$$

The approximated coefficients $a_{\alpha}$ must also satisfy the ellipticity condition

$$
\delta_{0} I_{m} \sum_{p=1}^{n} \xi_{p}^{2} \leq \sum_{p, q=1}^{n} a_{p, q, \alpha}(x, t) \xi_{p} \xi_{q} \leq \delta_{0}^{-1} I_{m} \sum_{p=1}^{n} \xi_{p}^{2}
$$

for some constant $\delta_{0}>0$ and arbitrary $\alpha \in(0,1), x \in \Omega, t \in I,\left(\xi_{1}, \ldots, \xi_{n}\right) \in R^{n}$.

We are interested in approximating the original data in the following spaces as $\alpha \rightarrow 0$,

$$
\begin{gathered}
f_{\alpha} \rightarrow f \quad \text { in } H^{s, L}(\Omega \times I), \quad \bar{g}_{\alpha} \rightarrow \bar{g}, \quad \text { in } H^{s+1, L}(\Omega \times I), \\
\partial a_{\alpha} \rightarrow \partial a \quad \text { in } H^{s, L-1}(\Omega \times I), \quad a_{\alpha} \rightarrow a \quad \text { in } H^{s+1, L}(\Omega \times I), \\
a_{\alpha}^{(j)}(0) \rightarrow a^{(j)}(0) \quad \text { in } H^{r_{j}+1}(\Omega), \quad j=0, \ldots, L-1, \\
u_{\alpha}^{j} \rightarrow u^{j} \quad \text { in } H^{r_{j}+2}(\Omega), \quad j=0, \ldots, L-1, \\
u_{\alpha}^{L} \rightarrow u^{L} \quad \text { in } H^{1}(\Omega) \cap H^{r_{L-2}}(\Omega), \\
u_{\alpha}^{L+1} \rightarrow u^{L+1} \quad \text { in } H^{r_{L-1}}(\Omega) .
\end{gathered}
$$

Theorem 2.3.1. If the conditions (2.2.3), (2.2.6)-(2.2.12) are fulfilled, then there exists a family (2.3.1) with the properties (2.3.2)-(2.3.7).

\subsection{Proof of the regularization theorem}

First consider the mollification $d_{\alpha}$ with respect to the $x, t$ variables of the function $a$. Let $a_{\alpha}^{j}$ be a family of smooth functions such that $a_{\alpha}^{j} \rightarrow a^{(j)}(0)$ as $\alpha \rightarrow 0$ in $H^{r_{j}+1}(\Omega), j=0, \ldots, L-1$. By the trace theory, cf. Lemma 5.2.2, Section 5.2 below, there exists a smooth function $e_{\alpha}$, such that $e_{\alpha}^{(j)}(0)=a_{\alpha}^{j}-d_{\alpha}^{(j)}(0)$ and

$$
\begin{gathered}
\left\|e_{\alpha}\right\|_{H^{s+1, L}(\Omega \times I)} \leq C \sum_{j=0}^{L-1}\left\|a_{\alpha}^{j}-d_{\alpha}^{(j)}(0)\right\|_{H^{s_{j}^{1}}(\Omega)}, \\
\left\|\partial e_{\alpha}\right\|_{H^{s, L-1}(\Omega \times I)} \leq C \sum_{j=0}^{L-2}\left\|a_{\alpha}^{j}-d_{\alpha}^{(j)}(0)\right\|_{H^{s_{j}^{2}+1}(\Omega)}, \\
s_{j}^{1}=\frac{s+1}{L}\left(L-j-\frac{1}{2}\right), \quad j=0, \ldots, L-1, \\
s_{j}^{2}=\frac{s}{L-1}\left(L-1-j-\frac{1}{2}\right), \quad j=0, \ldots, L-2 .
\end{gathered}
$$


If we put $a_{\alpha}=d_{\alpha}+e_{\alpha}$, then $a_{\alpha}^{(j)}(0)=a_{\alpha}^{j} \rightarrow a^{(j)}(0)$ in $H^{r_{j}+1}(\Omega), j=0, \ldots, L-1$ as $\alpha \rightarrow 0$. The continuity of the trace operator implies that $d_{\alpha}^{(j)}(0)-a^{(j)}(0) \rightarrow 0$ in $H^{s_{j}^{1}}(\Omega)$ and $H^{s_{j}^{2}+1}(\Omega)$ respectively as $\alpha \rightarrow 0$ for $j$ as before. As a consequence we obtain the convergence $\left\|e_{\alpha}\right\|_{H^{s+1, L}(\Omega)} \rightarrow 0,\left\|\partial e_{\alpha}\right\|_{H^{s, L-1}(\Omega)} \rightarrow 0$ as $\alpha \rightarrow 0$ and therefore the function $a_{\alpha}$ converges as in (2.3.5), (2.3.6). Applying mollifiers to the remaining data one can construct families

$$
\bar{f}_{\alpha}, \bar{g}_{\alpha}, a_{\alpha}, \bar{u}_{\alpha}^{j}, \quad j=0, \ldots, L+1, \quad \alpha \in(0,1),
$$

possessing the regularity as in (2.3.2) and converging as in (2.3.5)-(2.3.7). Since the approximated data (2.4.2) generally speaking do not satisfy the boundary and compatibility conditions, we have to improve them in an appropriate way. For this purpose let us define $u_{\alpha}^{0}$ as a solution of the boundary value problem

$$
\begin{gathered}
\bar{u}_{\alpha}^{2}+\gamma \bar{u}_{\alpha}^{0}=a_{\alpha}(0) \partial^{2} u_{\alpha}^{0}+\gamma u_{\alpha}^{0}+\bar{f}_{\alpha}(0) \text { in } \Omega, \\
b_{\alpha}(0) \partial u_{\alpha}^{0}=g_{\alpha}(0), \quad \text { on } \partial \Omega \text { for the Neumann b.c. } \\
u_{\alpha}^{0}=0, \quad \text { on } \partial \Omega \text { for Dirichlet b.c. }
\end{gathered}
$$

Note that from the inequality $r_{0}+1>n / 2$ it follows $H^{r_{0}+1} \subset C^{0}$, and $(2.3 .6)$ implies the convergence $a_{\alpha}(0) \rightarrow a(0)$ in $C^{0}(\Omega)$ as $\alpha \rightarrow 0$. Therefore, for sufficiently small $\alpha \in(0,1), a_{\alpha}(0) \partial^{2}$ satisfies the ellipticity condition (2.3.4) and one can choose a constant $\gamma>0$ such that the problem (2.4.3) is uniquely solvable in $C^{\infty}(\bar{\Omega})$.

Next, assuming that $u_{\alpha}^{0}, \ldots, u_{\alpha}^{j-1}$ are given, let us define $u_{\alpha}^{j}, j \leq L-1$ as the solution to the elliptic boundary value problem

$$
\begin{aligned}
& \bar{u}_{\alpha}^{j+2}+\gamma \bar{u}_{\alpha}^{j}=a_{\alpha}(0) \partial^{2} u_{\alpha}^{j}+\gamma u_{\alpha}^{j}+\bar{f}_{\alpha}^{(j)}(0) \\
& \quad+\sum_{i=1}^{j}\left(\begin{array}{l}
j \\
i
\end{array}\right) a_{\alpha}^{(i)}(0) \partial^{2} u_{\alpha}^{j-i}, \quad \text { in } \Omega, \\
& b_{\alpha}(0) \partial u_{\alpha}^{j}+\sum_{i=1}^{j}\left(\begin{array}{c}
j \\
i
\end{array}\right) b_{\alpha}^{(i)}(0) \partial u_{\alpha}^{j-i}=g_{\alpha}^{(j)}(0), \\
& \quad \text { on } \partial \Omega \text { for the Neumann b.c., } \\
& u_{\alpha}^{j}=0, \quad \text { on } \partial \Omega \text { for the Dirichlet b.c., }
\end{aligned}
$$

where $\gamma$ is a constant chosen in such a way, that both problems (2.4.3), (2.4.4) are uniquely solvable in $C^{\infty}(\bar{\Omega})$. Finally let us put

$$
u_{\alpha}^{j}=\bar{u}_{\alpha}^{j}, \quad j=L, L+1 .
$$

Now let $\widehat{f}_{\alpha}^{j}$ be the functions of the form

$$
\widehat{f}_{\alpha}^{j}=u_{\alpha}^{j+2}-\bar{u}_{\alpha}^{j+2}+\gamma\left(u_{\alpha}^{j}-\bar{u}_{\alpha}^{j}\right), \quad j=0, \ldots, L-1 .
$$


Since $\widehat{f}_{\alpha}^{j} \in C^{\infty}(\bar{\Omega})$, according to Lemma 5.2.2 from Section 5.2, there exists a function $\widehat{f}_{\alpha}$ with the properties

$$
\begin{gathered}
\widehat{f}_{\alpha} \in C^{\infty}(\bar{\Omega} \times \bar{I}), \quad \widehat{f}_{\alpha}^{(j)}(0)=\widehat{f}_{\alpha}^{j}, \quad j=0, \ldots, L-1, \quad \alpha \in(0,1), \\
\left\|\widehat{f}_{\alpha}\right\|_{H^{s, L}(\Omega \times I)} \leq c \sum_{j=0}^{L-1}\left\|\widehat{f}_{\alpha}^{j}\right\|_{H^{r_{j}}(\Omega)},
\end{gathered}
$$

for some positive constant $c$. If we define

$$
f_{\alpha}=\bar{f}_{\alpha}-\widehat{f}_{\alpha}
$$

then the corresponding family (2.3.1) will satisfy the compatibility condition (2.3.3).

It is sufficient to show that $f_{\alpha}$ and $u_{\alpha}^{j}$ satisfy (2.3.5), (2.3.7). Subtracting the equations (2.2.10), (2.2.11) and (2.4.4) side by side we obtain for $j=0, \ldots, L-1$,

$$
\begin{aligned}
\left(u^{j+2}-\bar{u}_{\alpha}^{j+2}\right)+\gamma\left(u^{j}-\bar{u}_{\alpha}^{j}\right)= & a_{\alpha}(0) \partial^{2}\left(u^{j}-u_{\alpha}^{j}\right)+\gamma\left(u^{j}-u_{\alpha}^{j}\right) \\
& +\left(a(0) \partial^{2}-a_{\alpha}(0) \partial^{2}\right) u^{j}+f^{(j)}(0)-\bar{f}_{\alpha}^{(j)}(0) \\
& +\sum_{i=1}^{j}\left(\begin{array}{c}
j \\
i
\end{array}\right)\left[a_{\alpha}^{(i)}(0) \partial^{2}\left(u^{j-i}-u_{\alpha}^{j-i}\right)\right. \\
& \left.+\left(a^{(i)}(0) \partial^{2}-a_{\alpha}^{(i)}(0) \partial^{2}\right) u^{j-i}\right], \quad \text { in } \Omega
\end{aligned}
$$

$$
\begin{gathered}
b_{\alpha}(0) \partial\left(u^{j}-u_{\alpha}^{j}\right)+\left(b(0) \partial-b_{\alpha}(0) \partial\right) u^{j} \\
+\sum_{i=1}^{j}\left(\begin{array}{c}
j \\
i
\end{array}\right)\left[b_{\alpha}^{(i)}(0) \partial\left(u^{j-i}-u_{\alpha}^{j-i}\right)+\left(b^{(i)}(0) \partial-b_{\alpha}^{(i)}(0) \partial\right) u^{j-i}\right] \\
=g^{(j)}(0)-g_{\alpha}^{(j)}(0) \text { on } \partial \Omega, \text { for the Neumann b.c., } \\
u_{\alpha}^{j}=0 \text { on } \partial \Omega, \text { for the Dirichlet b.c. }
\end{gathered}
$$

Since for $p, \theta=2$ the Besov spaces $B_{p, \theta}^{s}$ reduce to the Sobolev spaces $H^{s}$ (cf. [24], Sect. 9.3), the elliptic estimate (6.1.1) from Section 6.1 gives for $j=0, \ldots, L-1$,

$$
\left\|u^{j}-u_{\alpha}^{j}\right\|_{H^{r_{j}+2}(\Omega)} \leq c\left(\delta_{0},\left\|a_{\alpha}(0)\right\|_{H^{r_{0}+1}(\Omega)}\right)\left(F_{j, \alpha}+G_{j, \alpha}\right)
$$


where

$$
\begin{aligned}
F_{j, \alpha} & =\sum_{i=1}^{6}\left\|F_{j, \alpha}^{i}\right\|_{H^{r} j(\Omega)} \\
F_{j, \alpha}^{1} & =u^{j+2}-\bar{u}_{\alpha}^{j+2}, \quad F_{j, \alpha}^{2}=\gamma\left(u^{j}-\bar{u}_{\alpha}^{j}\right), \\
F_{j, \alpha}^{3} & =\left(a(0) \partial^{2}-a_{\alpha}(0) \partial^{2}\right) u^{j}, \\
F_{j, \alpha}^{4} & =\sum_{i=1}^{j}\left(\begin{array}{l}
j \\
i
\end{array}\right) a_{\alpha}^{(i)}(0) \partial^{2}\left(u^{j-i}-u_{\alpha}^{j-i}\right), \\
F_{j, \alpha}^{5} & =\sum_{i=1}^{j}\left(\begin{array}{l}
j \\
i
\end{array}\right)\left(a^{(i)}(0) \partial^{2}-a_{\alpha}^{(i)}(0) \partial^{2}\right) u^{j-i}, \\
F_{j, \alpha}^{6} & =f^{(j)}(0)-\bar{f}_{\alpha}^{(j)}(0), \\
G_{j, \alpha} & =\sum_{i=1}^{4}\left\|G_{j, \alpha}^{i}\right\|_{H^{r_{j}+1-1 / 2}(\partial \Omega)}, \quad \text { for the Neumann b.c. }, \\
G_{j, \alpha} & =0, \quad \text { for the Dirichlet b.c. }, \\
G_{j, \alpha}^{1} & =\left(b(0) \partial-b_{\alpha}(0) \partial\right) u^{j}, \\
G_{j, \alpha}^{2} & =\sum_{i=1}^{j}\left(\begin{array}{l}
j \\
i
\end{array}\right) b_{\alpha}^{(i)}(0) \partial\left(u^{j-i}-u_{\alpha}^{j-i}\right), \\
G_{j, \alpha}^{3}= & \sum_{i=1}^{j}\left(\begin{array}{l}
j \\
i
\end{array}\right)\left(b^{(i)}(0) \partial-b_{\alpha}^{(i)}(0) \partial\right) u^{j-i}, \\
G_{j, \alpha}^{4}= & g^{(j)}(0)-g_{\alpha}^{(j)}(0) .
\end{aligned}
$$

Using Lemmas 5.3.2 and 5.3.3 we can check that $F_{j, \alpha}, G_{j, \alpha}$ converge to 0 as $\alpha \rightarrow 0$, $j=0, \ldots, L-1$ and consequently $u_{\alpha}^{j}$ satisfies (2.3.7). To prove that $f_{\alpha}$ satisfies (2.3.5) we have to check that $\widehat{f}_{\alpha} \rightarrow 0$ in $H^{s, L}(\Omega \times I)$ as $\alpha \rightarrow 0$. According to (2.4.8) it is sufficient to prove that $\widehat{f}_{\alpha}^{j} \rightarrow 0$ in $H^{r_{j}}(\Omega), j=0, \ldots, L-1$ and the last relation is a consequence of (2.4.6), (2.3.7) and Lemma 5.3.4.

\section{Energy estimates for non-smooth Cauchy-Dirichlet problem}

\subsection{Introductory remarks}

The energy estimates for hyperbolic mixed problems play a fundamental role in the existence theorems in both the linear and the nonlinear case. In the latter case, the regularity of coefficients depends on the regularity of solutions. Since the solutions are usually assumed to be functions of $t \in I \subset R$ with values in some 
Sobolev space $H^{s}(\Omega), \Omega \subset R^{n}, s \in R$, it is natural to describe the regularity of coefficients in the same language.

The first multidimensional hyperbolic estimates, applicable to the quasilinear mixed problems of the second order, were obtained in [18]. Contemporary results are much more optimal. For example in [8] and [15] the norm

$$
\max _{t \in I} \sum_{k=0}^{L+1}\left\|\partial_{t}^{k} u(t)\right\|_{H^{L+1-k}(\Omega)}, \quad L \geq[n / 2]+2, \quad L \text { - integer }
$$

of the solution to the linear, second order Cauchy-Dirichlet problem was estimated by the data and a constant depending on the norm

$$
\underset{t \in I}{\operatorname{ess} \sup } \sum_{k=0}^{L}\left\|\partial_{t}^{k} a(t)\right\|_{H^{L-k}(\Omega)}
$$

of the coefficients $a=\left\{a_{p, q}: p, q=1, \ldots, n+1\right\}$, cf. (3.2.2). From the embedding theorems it follows that the coefficients with finite norm (3.1.2) are Lipschitzcontinuous on $\Omega \times I$ and the solutions with finite norm (3.1.1) are the classical ones, i.e. they possess the second derivatives continuous on $\bar{\Omega} \times \bar{I}$. To include the nonclassical solutions into considerations it is necessary to investigate the linear problems with coefficients less regular than those in $[8,15]$.

In Section 2 we have proved that some class of such nonregular problems can be approximated by the smooth ones. Now we are interested in an appropriate energy estimate. In (3.3.3) below we formulate precisely what kind of the estimate we have in mind. It is important that in our approach it is sufficient to assume that the following norm

$$
|a|^{2}=\int_{I}\left(\|a(t)\|_{H^{\bar{s}+1}(\Omega)}^{2}+\left\|\partial_{t}^{L-1} a(t)\right\|_{H^{1}(\Omega)}^{2}+\left\|\partial_{t}^{L} a(t)\right\|_{H^{0}(\Omega)}^{2}\right) d t
$$

of coefficients $a=\left\{a_{p, q}\right\}$ is finite, where $\bar{s} \in R, n / 2<\bar{s} \leq L-1$.

One can easily see that the functions with finite norm (3.1.3) are not necessarily Lipschitz-continuous on $\Omega \times I$. If the assumptions of both the present section and Section 2 are satisfied, then using (3.3.3) it is possible to prove that the sequence of smooth solutions corresponding to all regularized problems from Section 2 is uniformly bounded in some sufficiently strong norm. Consequently, one can obtain a solution to the nonregularized problem as a weak limit of solutions to the regularized one. The corresponding argument is given in Section 4. From the form of the inequality (3.3.3) it follows that the obtained solution is not necessarily the classical one. The relaxation of the regularity is possible due the fact that in the proof of (3.3.3), uniformly with respect to $t \in I$, elliptic estimates are replaced by the corresponding estimates with the $L^{2}$ dependence on $t$, (cf. Section 6 below). To 
overcome some technical difficulties connected with this approach, we also replace the usual norms of the solution by the weighted ones, cf. (3.3.1), (3.3.3).

\subsection{Basic assumptions}

Let us consider the linear, initial-boundary value problem

$$
\begin{array}{ll}
u^{(2)}=a \partial^{2} u+f, & \text { in } \Omega \times I, \\
u=0, & \text { on } \partial \Omega \times I, \\
u(0)=u^{0}, \quad u^{(1)}(0)=u^{1}, & \text { on } \Omega .
\end{array}
$$

Here we use the same notations as in Section 2.2, and the operator $a \partial^{2}$ is defined as before:

$$
a \partial^{2} u=\sum_{p, q=1}^{n+1} a_{p, q} \partial_{p} \partial_{q} u .
$$

We shall assume that real numbers $s, \bar{s}$ and an integer $L$ are given which satisfy the following relations:

$$
\begin{aligned}
& \quad \frac{n}{2}<\bar{s}<s \leq L-1, \quad s-\bar{s} \leq \frac{1}{2} \\
& s_{j}+\frac{3}{2} \text { are nonintegers for } j=0, \ldots, L-1, \\
& \quad \text { where } s_{j}=\frac{s}{L-1}(L-1-j) .
\end{aligned}
$$

Let the conditions $(2.2 .6),(2.2 .8),(2.2 .10),(2.2 .12)$ from Section 2.2 be fulfilled and additionally

$$
\begin{aligned}
u^{j} \in H_{0}^{1}(\Omega) \cap H^{r_{j}+2}(\Omega), & j=0, \ldots, L-1, \\
u^{L} \in H_{0}^{1}(\Omega) \cap H^{r_{L-2}}(\Omega), & u^{L+1} \in H^{r_{L-1}}(\Omega) .
\end{aligned}
$$

Here $u^{2}, \ldots, u^{L+1}$ are defined with the use of the recursive formula (2.2.10) and the space $H_{0}^{1}(\Omega)$ is defined as follows

$$
H_{0}^{1}(\Omega)=\left\{u \in H^{1}(\Omega): u=0 \text { on } \partial \Omega \text { in the sense of the trace }\right\} .
$$

REMARK. The assumption (3.2.4) contains both the regularity and the compatibility conditions.

\subsection{The basic estimate}

Let us assume that $\lambda \geq 0$ is a constant, $t \in I$ and introduce the following notations. 


$$
\begin{aligned}
& (u(t), v(t))_{0, \lambda}=\int_{\Omega} u(t) v(t) e^{-2 \lambda t} d x, \\
& (u, v)_{0, \lambda, t}=\int_{0}^{t}(u(\tau), v(\tau))_{0, \lambda} d \tau \\
& |v(t)|_{0, \lambda}^{2}=(v(t), v(t))_{0, \lambda}, \\
& |v|_{r} \text {-the norm of the Sobolev space } H^{r}(\Omega)=H^{r}, \\
& |v(t)|_{r, \lambda}^{2}=|v(t)|_{r}^{2} e^{-2 \lambda t}, \\
& |v|_{0,1, \lambda}^{2}=\left|v^{(1)}\right|_{0, \lambda}^{2}+|v|_{1, \lambda}^{2}, \\
& |v|_{0,1, \lambda, t}^{2}=\int_{0}^{t}|v(\tau)|_{0,1, \lambda}^{2} d \tau, \\
& \|v\|_{0,1, \lambda, t}^{2}=\operatorname{ess~sup}_{\tau \in(0, t)}|v(\tau)|_{0,1, \lambda}^{2}, \\
& \|v\|_{r, \lambda, t}^{2}=\operatorname{ess~sup}_{\tau \in(0, t)}|v(\tau)|_{r, \lambda}^{2}, \\
& |v|_{r, \lambda, t}^{2}=\int_{0}^{t}|v(\tau)|_{r, \lambda}^{2} d \tau, \\
& \left\|u^{j}\right\|_{0,1}^{2}=\left|u^{j+1}\right|_{0}^{2}+\left|u^{j}\right|_{1}^{2}, \quad j=0, \ldots, L .
\end{aligned}
$$

Let us recall that the symbols $L, s_{k}$ and $\delta$ were introduced in (3.2.3) and (2.2.12) respectively. Let us introduce the norm of coefficients which is a little weaker than that introduced in the formula (3.1.3), (cf. Section 5.1)

$$
\begin{aligned}
\|a\|^{2}= & \max _{t \in I}|a(t)|_{\bar{s}_{0}^{1}+1}^{2}+\underset{t \in I}{\operatorname{ess} \sup }\left(\sum_{j=1}^{L-2}\left|a^{(j)}(t)\right|_{\bar{s}_{j}^{1}+1}^{2}+\left|a^{(L-1)}(t)\right|_{\bar{s}_{L-1}^{2}}^{2}\right) \\
& +\int_{I}\left\{\sum_{j=0}^{L-2}\left|a^{(j)}(t)\right|_{\bar{s}_{j}+1}^{2}+\left|a^{(L-1)}(t)\right|_{\bar{s}_{L-2}}^{2}+\left|a^{(L)}(t)\right|_{0}^{2}\right\} d t, \\
\bar{s}_{j}= & \frac{\bar{s}}{L-1}(L-1-j), \quad \bar{s}_{j}^{1}=\frac{\bar{s}}{L-1}\left(L-1-j-\frac{1}{2}\right), \\
\bar{s}_{j}^{2}= & \frac{\bar{s}+1}{L}\left(L-j-\frac{1}{2}\right) .
\end{aligned}
$$

The main purpose of the present section is to prove the following estimate.

Theorem 3.3.1. If the assumptions (2.2.6), (2.2.8), (2.2.12), (3.2.3), (3.2.4) are satisfied then there exist positive constants $C=C(\delta)$ and $\lambda_{0}=\lambda_{0}(\delta,\|a\|)$, such 
that the inequality

$$
\begin{aligned}
& \sum_{0 \leq j \leq L} \lambda^{2 j}\left(\left\|u^{(L-j)}\right\|_{0,1, \lambda, t}^{2}+\lambda\left|u^{(L-j)}\right|_{0,1, \lambda, t}^{2}\right) \\
& +\lambda \sum_{0 \leq l \leq k \leq L-1} \lambda^{2 l}\left|u^{(k-l)}\right|_{s_{k}+2, \lambda, t}^{2} \\
& \leq C(\delta)\left\{\sum_{0 \leq j \leq L} \lambda^{2 j}\left[\left\|u^{L-j}\right\|_{0,1}^{2}+\left(\int_{0}^{t}\left|f^{(L-j)}(\tau)\right|_{0} e^{-\lambda \tau} d \tau\right)^{2}\right]\right. \\
& \left.+\sum_{0 \leq l \leq k \leq L-1} \lambda^{2 l+1}\left|f^{(k-l)}\right|_{s_{k}, \lambda, t}^{2}\right\}
\end{aligned}
$$

holds true for arbitrary $\lambda \geq \lambda_{0}$ and arbitrary $u$ satisfying (3.2.1) and the relations

$$
\begin{aligned}
& u^{(j+1)} \in L^{\infty}\left(I, H^{0}(\Omega)\right), \\
& u^{(j)} \in L^{\infty}\left(I, H_{0}^{1}(\Omega)\right), \\
& u^{(j)} \in L^{2}\left(I, H^{s_{j}+2}(\Omega) \cap H_{0}^{1}(\Omega)\right), \\
& \quad j=0, \ldots, L-1 .
\end{aligned}
$$

\subsection{First step of the proof}

Assume temporarily that $u$ is arbitrarily smooth with respect to $t$ and differentiate the equation

$$
u^{(2)}-a \partial^{2} u+\gamma u=f+\gamma u
$$

$L-j$ times, $0 \leq j \leq L$ with respect to $t$. Here $\gamma$ denotes some constant to be determined later, cf. (3.4.6). Multiplying both sides by $u^{(L-j+1)} e^{-2 \lambda t}$ and integrating over $\Omega$, we obtain

$$
\begin{aligned}
\left(u^{(L-j+2)}, u^{(L-j+1)}\right)_{0, \lambda}- & \left(a \partial^{2} u^{(L-j)}, u^{(L-j+1}\right)_{0, \lambda} \\
+\gamma\left(u^{(L-j)}, u^{(L-j+1)}\right)_{0, \lambda}= & \left(f^{(L-j)}+F_{1}^{L-j}, u^{(L-j+1)}\right)_{0, \lambda} \\
& +\gamma\left(u^{(L-j)}, u^{(L-j+1)}\right)_{0, \lambda}, \quad 0 \leq j \leq L,
\end{aligned}
$$

where

$$
F_{1}^{L-j}=\sum_{i=1}^{L-j}\left(\begin{array}{c}
L-j \\
i
\end{array}\right) a^{(i)} \partial^{2} u^{(L-j-i)}, \quad 0 \leq j \leq L-1, \quad F_{1}^{0}=0 .
$$

Integrating (3.4.2) over $(0, t)$ and using the formula

$$
\frac{d}{d t}\left\{(v, v)_{0, \lambda}\right\}=-2 \lambda(v, v)_{0, \lambda}+2\left(v, v^{(1)}\right)_{0, \lambda},
$$


we get for $0 \leq j \leq L$

$$
\begin{aligned}
& \left|u^{(L-j+1)}(t)\right|_{0, \lambda}^{2}-\left|u^{(L-j+1)}(0)\right|_{0}^{2}+2 \lambda\left|u^{(L-j+1)}\right|_{0, \lambda, t}^{2} \\
& \quad+a_{\gamma}\left(\partial u^{(L-j)}(t), \partial u^{(L-j)}(t)\right)-a_{\gamma}\left(\partial u^{(L-j)}(0), \partial u^{(L-j)}(0)\right) \\
& +2 \lambda \int_{0}^{t} a_{\gamma}\left(\partial u^{(L-j)}(\tau), \partial u^{(L-j)}(\tau)\right) d \tau \\
& \quad=2\left(f^{(L-j)}+F_{1}^{L-j}, u^{(L-j+1)}\right)_{0, \lambda, t}+F_{2, t}^{L-j}\left(u^{(L-j)}, u^{(L-j)}\right) .
\end{aligned}
$$

Here

$$
\begin{gathered}
a_{\gamma}(\partial v, \partial v)=\sum_{p, q=1}^{n}\left(a_{p, q} \partial_{q} v, \partial_{p} v\right)_{0, \lambda}+\gamma(v, v)_{0, \lambda} \\
F_{2, t}^{j}\left(u^{(j)}, u^{(j)}\right)=\sum_{p, q=1}^{n}\left(a_{p, q}^{(1)} \partial_{q} u^{(j)}, \partial_{p} u^{(j)}\right)_{0, \lambda, t}+\gamma\left(u^{(j)}, u^{(j+1)}\right)_{0, \lambda, t} \\
+\sum_{p, q=1}^{n}\left(\left(\partial_{p} a_{p, q}\right) \partial_{q} u^{(j)}, u^{(j+1)}\right)_{0, \lambda, t} \\
+\sum_{\substack{p=n+1 \\
\text { or } q=n+1}}\left(a_{p, q} \partial_{p} \partial_{q} u^{(j)}, u^{(j+1)}\right)_{0, \lambda, t}
\end{gathered}
$$

and $\gamma=\gamma(\delta,\|a\|)$ is an appropriately chosen constant so that

$$
a_{\gamma}(\partial v, \partial v) \geq \frac{1}{2} \delta|\partial v|_{0}^{2} \quad \text { for arbitrary } v \in H_{0}^{1}(\Omega) .
$$

From (3.4.4) and (3.4.6) we deduce

$$
\begin{gathered}
\left|u^{(L-j)}(t)\right|_{0,1, \lambda}^{2}+2 \lambda\left|u^{(L-j)}\right|_{0,1, \lambda, t}^{2} \\
\leq C_{j}(\delta)\left\{\left\|u^{L-j}\right\|_{0,1}^{2}+2\left(f^{(L-j)}+F_{1}^{L-j}, u^{(L-j+1)}\right)_{0, \lambda, t}\right. \\
\left.\quad+F_{2, t}^{L-j}\left(u^{(L-j)}, u^{(L-j)}\right)\right\}, \quad 0 \leq j \leq L .
\end{gathered}
$$

Now, since (3.4.7) makes sense for $u$ satisfying (3.3.4), using mollifiers with respect to $t$ and Lemmas 5.5.1, 5.5.2 from Section 5.5 below, we can drop the additional regularity assumption concerning $u$. To be more precise, applying the smoothing operation from Section 5.5 to both sides of (3.4.1) and repeating the previous argument we can obtain a relation analogous to (3.4.7), with $u$ replaced by $u_{\sigma}$ and $f$ replaced by $f_{\sigma}+I_{\sigma}$. Here we use the same notations as in Section 5.5 with $v=\partial^{2} u$. The estimates from the remaining part of the present section, combined with Lemmas 5.5.1, 5.5.2 show that if $\sigma \rightarrow 0$ then we obtain (3.4.7) for $u$ satisfying (3.3.4). 
Multiplying both sides of (3.4.7) by $\lambda^{2 j}$ and summing over $0 \leq j \leq L$ we get

$$
E_{1}^{t}+2 \lambda E_{2}^{t} \leq C_{1} \sum_{k=3}^{6} E_{k}^{t},
$$

with some positive constant $C_{1}$ and

$$
E_{k}^{t}=\sum_{0 \leq j \leq L} \lambda^{2 j} e_{k, L-j}^{t}, \quad k=1, \ldots, 6,
$$

where

$$
\begin{array}{ll}
e_{1, j}^{t}=\left\|u^{(j)}\right\|_{0,1, \lambda, t}^{2}, & e_{2, j}^{t}=\left|u^{(j)}\right|_{0,1, \lambda, t}^{2}, \\
e_{3, j}^{t}=\left\|u^{j}\right\|_{0,1}^{2}, & e_{4, j}^{t}=2\left(f^{(j)}, u^{(j+1)}\right)_{0, \lambda}, \\
e_{5, j}^{t}=2\left(F_{1}^{j}, u^{(j+1)}\right)_{0, \lambda}, & e_{6, j}^{t}=F_{2, t}^{j}\left(u^{(j)}, u^{(j)}\right) .
\end{array}
$$

The term $E_{4}^{t}$ can be estimated without difficulties:

$$
C_{1} E_{4}^{t} \leq c_{0} E_{7}^{t}+4^{-1} E_{1}^{t}
$$

where $E_{7}^{t}$ is defined as in (3.4.9) with $k=7$ and

$$
e_{7, L-j}^{t}=\left(\int_{0}^{t}\left|f^{(L-j)}\right|_{0} e^{-\lambda \tau} d \tau\right)^{2} .
$$

Next let us note that from Lemma 5.1.1, Sect. 5.1 below it follows that if $\partial a \in L^{2}\left(I, H^{\bar{s}}(\Omega)\right), \partial a^{(L-1)} \in L^{2}\left(I, H^{0}(\Omega)\right)$, then for some constant $C>0$,

$$
\begin{aligned}
& a^{(i)} \in L^{2}\left(I, H^{\bar{s}_{i}+1}(\Omega)\right), \quad i=0, \ldots, L-1, \\
& \quad \text { with } \bar{s}_{i}=\frac{\bar{s}}{L-1}(L-1-i), \\
& \sum_{i=0}^{L-1}\left\|a^{(i)}\right\|_{L^{2}\left(I, H^{\bar{s}_{i}+1}(\Omega)\right)} \\
& \quad \leq C\left(\|a\|_{L^{2}\left(I, H^{\bar{s}+1}(\Omega)\right)}+\left\|a^{(L-1)}\right\|_{L^{2}\left(I, H^{1}(\Omega)\right)}\right) .
\end{aligned}
$$

Similarly, if $\partial^{2} u \in L^{2}\left(I, H^{s}(\Omega)\right), \partial^{2} u^{(L-1)} \in L^{2}\left(I, H^{0}(\Omega)\right)$, where $\partial^{2}=\left\{\partial^{|\alpha|}:|\alpha|=\right.$ $2\}$, then

$$
u^{(i)} \in L^{2}\left(I, H^{s_{i}+2}(\Omega)\right), \quad i=0, \ldots, L-1, \quad s_{i}=\frac{s}{L-1}(L-1-i),
$$

$$
\begin{aligned}
& \sum_{i=0}^{L-1}\left\|u^{(i)}\right\|_{L^{2}\left(I, H^{s_{i}+2}(\Omega)\right)} \\
& \quad \leq C\left(\|u\|_{L^{2}\left(I, H^{s+2}(\Omega)\right)}+\left\|u^{(L-1)}\right\|_{L^{2}\left(I, H^{2}(\Omega)\right)}\right),
\end{aligned}
$$


for some constant $C>0$. The relations

$$
\begin{gathered}
\bar{s}_{i}+1+s_{L-i}>\bar{s}+1-\frac{s}{L-1} \geq \bar{s}>\frac{n}{2}, \\
\bar{s}_{i} \geq 0, \quad s_{L-i} \geq 0, \\
1 \leq i \leq L-j, \quad 0 \leq j \leq L-1, \quad i \leq L-2, \\
\bar{s}_{L-2}+s_{1}>\bar{s}>\frac{n}{2},
\end{gathered}
$$

combined with Lemmas 5.3.1, 5.3.5 below, imply

$$
\begin{aligned}
&\left|a^{(i)} \partial^{2} u^{(L-j-i)}\right|_{0} \leq c\left|a^{(i)}\right|_{\bar{s}_{i}+1}\left|u^{(L-j-i)}\right|_{s_{L-i}+2}, i, j \text { as in }(3.4 .15), \\
&\left.i a^{(L-1)} \partial^{2} u^{(1-j)}\right|_{0} \leq c\left|a^{(L-1)}\right|_{\bar{s}_{L-2}}\left|u^{(1-j)}\right|_{s_{1}+2}, \quad j=0,1, \\
&\left|a^{(L)} \partial^{2} u\right|_{0} \leq c\left|a^{(L)}\right|_{0}|u|_{s+2} .
\end{aligned}
$$

As a consequence we obtain the following estimate for $E_{5}^{t}$

$$
\begin{aligned}
C_{1} E_{5}^{t} & \leq c_{0}\|a\|^{2} E_{8}^{t}+4^{-1} E_{1}^{t}, \quad(\|a\| \text { is defined in (3.3.2)), } \\
E_{8}^{t} & =\sum_{\substack{0 \leq j \leq L-1 \\
1 \leq i \leq L-j}} \lambda^{2 j}\left|u^{(L-j-i)}\right|_{s_{L-i}+2, \lambda, t}^{2}+|u|_{s+2, \lambda, t}^{2} .
\end{aligned}
$$

To estimate the three-linear terms contained in $E_{6}^{t}$, one can use the inequalities

$$
\begin{aligned}
\int_{0}^{t}(b v, w)_{0, \lambda} d \tau & \leq|b|_{L^{2}\left(I, H^{r}(\Omega)\right)}|v|_{0, \lambda, t}\|w\|_{0, \lambda, t} \\
& \leq c|b|_{L^{2}\left(I, H^{r}(\Omega)\right)}^{2}|v|_{0, \lambda, t}^{2}+4^{-1}\|w\|_{0, \lambda, t}^{2}, \quad\left(r>\frac{n}{2}\right),
\end{aligned}
$$

which imply

$$
C_{1} E_{6}^{t} \leq c_{0}(\|a\|+\gamma)^{2} E_{2}^{t}+4^{-1} E_{1}^{t} .
$$

Combining (3.4.8), (3.4.11), (3.4.17) and (3.4.19) we see that if $\lambda$ is sufficiently large so as to satisfy

$$
c_{0}(\|a\|+\gamma)^{2} \leq \lambda
$$

then

$$
4^{-1} E_{1}^{t}+\lambda E_{2}^{t} \leq C^{1}(\delta)\left(E_{3}^{t}+E_{7}^{t}+\|a\|^{2} E_{8}^{t}\right)
$$

for some positive constant $C^{1}(\delta)$ independent of $u$ and $a$. 


\subsection{Application of elliptic estimates with $L^{2}$ dependence on $t$}

Now we shall use the ellipticity of the operator $a \partial^{2}$. For this purpose let us differentiate the equation

$$
a \partial^{2} u=u^{(2)}-f
$$

$k-l$ times with respect to $t$, where $0 \leq l \leq k \leq L-1$. We obtain

$$
\begin{aligned}
& a \partial^{2} u^{(k-l)}=u^{(k-l+2)}-f^{(k-l)}-F_{1}^{k-l}, \\
& F_{1}^{k-l}=\sum_{i=1}^{k-l}\left(\begin{array}{c}
k-l \\
i
\end{array}\right) a^{(i)} \partial^{2} u^{(k-l-i)}, \quad \text { if } 0 \leq l<k \leq L-1, \\
& F_{1}^{k-l}=0, \quad \text { if } l=k .
\end{aligned}
$$

In the special case $p=\theta=2$ the Besov spaces $B_{p, \theta}^{s}$ reduce to the Sobolev spaces $H^{s}$, cf. [24], Sect. 9.3, so the estimate (6.2.1) from Section 6.2 implies

$$
\begin{array}{r}
\left|u^{(k-l)}\right|_{s_{k}+2, \lambda, t}^{2} \leq C_{l}^{k}\left\{\left|u^{(k-l+2)}\right|_{s_{k}, \lambda, t}^{2}+\left|f^{(k-l)}\right|_{s_{k}, \lambda, t}^{2}\right. \\
\left.+\left|F_{1}^{k-l}\right|_{s_{k}, \lambda, t}^{2}+\bar{C}_{l}^{k}(\|a\|)\left|u^{(k-l)}\right|_{0, \lambda, t}^{2}\right\} \\
\text { for } 0 \leq l \leq k \leq L-1
\end{array}
$$

and for some constants $C_{l}^{k}=C_{l}^{k}(\delta)>0, \bar{C}_{l}^{k}(\|a\|)>0$. Note that from the relation (3.5.12), below it follows $a \in C^{0}\left(I, H^{\bar{s}_{0}^{1}+1}(\Omega)\right)$. The relations $\bar{s}_{0}^{1}+1=\bar{s}+1-\frac{1}{2} \frac{\bar{s}}{L-1} \geq$ $\bar{s}+\frac{1}{2} \geq s_{0}=s>\frac{n}{2}$, imply that we can apply Lemma 6.2.2 with $s^{0}=\bar{s}_{0}^{1}$ and $s=s_{k}$, $k=0, \ldots, L-1$.

Multiplying both sides of (3.5.3) by $\varepsilon_{l}^{k} \lambda^{2 l+1}$, where $\varepsilon_{l}^{k}$ are some constants satisfying the inequalities $0<\varepsilon_{l}^{k} \leq 1$, and summing side by side we get

$$
G_{1}^{t} \leq \sum_{m=2}^{7} G_{m}^{t},
$$

where

$$
\begin{aligned}
G_{m}^{t} & =\lambda \sum_{0 \leq l \leq k \leq L-1} \lambda^{2 l} g_{m, k, l}^{t}, \quad m=1,5,6,7, \\
G_{2}^{t} & =\lambda \sum_{0 \leq l \leq L-1} \lambda^{2 l} g_{2, L-1, l}^{t}, \quad(k=L-1), \\
G_{3}^{t} & =\lambda \sum_{0 \leq l \leq L-2} \lambda^{2 l} g_{3, L-2, l}^{l}, \quad(k=L-2),
\end{aligned}
$$




$$
\begin{aligned}
& G_{4}^{t}=\lambda \sum_{0 \leq l \leq k \leq L-3} \lambda^{2 l} g_{4, k, l}^{t}, \quad(k \leq L-3), \\
& g_{1, k, l}^{t}=\varepsilon_{l}^{k}\left|u^{(k-l)}\right|_{s_{k}+2, \lambda, t}^{2} \\
& g_{2, L-1, l}^{t}=\varepsilon_{1}^{L-1} C_{l}^{L-1}\left|u^{(L+1-l)}\right|_{s_{L-1}, \lambda, t,}^{2} \\
& g_{3, L-2, l}^{t}=\varepsilon_{l}^{L-2} C_{l}^{L-2}\left|u^{(L-l)}\right|_{s_{L-2}, \lambda, t}^{2}, \\
& g_{4, k, l}^{t}=\varepsilon_{l}^{k} C_{l}^{k}\left|u^{(k-l+2)}\right|_{s_{k}, \lambda, t}^{2} \\
& g_{5, k, l}^{t}=\varepsilon_{l}^{k} C_{l}^{k}\left|f^{(k-l)}\right|_{s_{k}, \lambda, t}^{2}, \\
& g_{6, k, l}^{t}=\varepsilon_{l}^{k} C_{l}^{k} \bar{C}_{l}^{k}(\|a\|)\left|u^{(k-l)}\right|_{0, \lambda, t}^{2}, \\
& g_{7, k, l}^{t}=\varepsilon_{l}^{k} C_{l}^{k}\left|F_{1}^{k-l}\right|_{s_{k}, \lambda, t}^{2} .
\end{aligned}
$$

Observe, that since $s_{L-1}=0, s_{L-2} \leq 1$, the terms $G_{2}^{t}, G_{3}^{t}$, can be estimated as follows

$$
G_{2}^{t}+G_{3}^{t} \leq 4^{-1} \lambda E_{2}^{t}
$$

provided

$$
\varepsilon_{l}^{k} C_{l}^{k} \leq 4^{-1}, \quad k=L-2, L-1, \quad 0 \leq l \leq k .
$$

If we assume that the constants $\varepsilon_{l}^{k}, C_{l}^{k}$ satisfy the condition

$$
\varepsilon_{l}^{k} C_{l}^{k} \leq 4^{-1} \varepsilon_{l}^{k+2} C_{l}^{k+2}, \quad 0 \leq l \leq k \leq L-3,
$$

then

$$
G_{4}^{t} \leq 4^{-1} G_{1}^{t}
$$

To estimate $G_{6}^{t}$, let us assume that $\lambda$ is sufficiently large to satisfy

$$
\varepsilon_{l}^{k} C_{l}^{k} \bar{C}_{l}^{k}(\|a\|) \leq 4^{-1} \lambda^{2(L-(k-l))}, \quad 0 \leq l \leq k \leq L-1 .
$$

We obtain

$$
G_{6}^{t} \leq 4^{-1} \lambda E_{2}^{t}
$$

Lemma 5.1.2 implies, that if we have $\partial a \in L^{2}\left(I, H^{\bar{s}}(\Omega)\right), \partial a^{(L-1)} \in L^{2}\left(I, H^{0}(\Omega)\right)$, then

$$
\begin{gathered}
a^{(j)} \in C^{0}\left(I, H^{\bar{s}_{j}^{1}+1}(\Omega)\right), \\
\bar{s}_{j}^{1}=\frac{\bar{s}}{L-1}\left(L-1-j-\frac{1}{2}\right), \quad j=0, \ldots, L-2, \\
\sum_{j=0}^{L-2}\left\|a^{(j)}\right\|_{C^{0}\left(I, H^{\bar{s}_{j}^{1}+1}(\Omega)\right)} \leq c\|a\|,
\end{gathered}
$$


for some positive constant $c$.

Using Lemma 5.3.1 we also can check that the inequalities

$$
\begin{aligned}
& \bar{s}_{i}^{1}+1+s_{k-i}^{1}-s_{k}>\bar{s}-\frac{s}{L-1}+1 \geq \bar{s}>\frac{n}{2} \\
& \bar{s}_{i}^{1}+1-s_{k}>\bar{s}-s+1-\frac{1}{2} \frac{\bar{s}}{L-1} \geq 0 \\
& s_{k-i}^{1}-s_{k}=\frac{s}{L-1}\left(i-\frac{1}{2}\right) \geq 0 \\
& s_{j}^{1}=\frac{s}{L-1}\left(L-1-j-\frac{1}{2}\right), \quad 0 \leq l<k \leq L-1, \\
& 1 \leq i \leq k-l, \quad i \leq L-2,
\end{aligned}
$$

imply for $i \leq L-2$

$$
\begin{aligned}
\left|a^{(i)} \partial^{2} u^{(k-l-i)}\right|_{s_{k}, \lambda, t}^{2} & \leq c\left|a^{(i)}\right|_{\bar{s}_{i}^{1}+1}^{2} \sum_{i=1}^{k-l}\left|\partial^{2} u^{(k-l-i)}\right|_{s_{k-i}^{1}, \lambda, t}^{2} \\
0 & \leq l<k \leq L-1
\end{aligned}
$$

Applying the interpolation inequality

$$
|v|_{\sigma_{1}(1-\theta)+\sigma_{2} \theta} \leq c|v|_{\sigma_{1}}^{1-\theta}|v|_{\sigma_{2}}^{\theta}
$$

with $\theta=\frac{1}{2}, \sigma_{1}=s_{j}, \sigma_{2}=s_{j+1}$ and using the fact that $\frac{1}{2} s_{j}+\frac{1}{2} s_{j+1}=s_{j}^{1}$, we can obtain the estimate

$$
|v|_{s_{j}^{1}}^{2} \leq c|v|_{s_{j}}|v|_{s_{j+1}} \leq c\left(\lambda^{-1}|v|_{s_{j}}^{2}+\lambda|v|_{s_{j+1}}^{2}\right)
$$

If $i=L-1$ then the relations $a^{(L)} \in L^{2}\left(I, H^{0}(\Omega)\right), a \in L^{2}\left(I, H^{\bar{s}+1}(\Omega)\right)$ give

$$
\begin{gathered}
a^{(j)} \in C^{0}\left(I, H^{\bar{s}_{j}^{2}}(\Omega)\right), \\
\bar{s}_{j}^{2}=\frac{\bar{s}+1}{L}\left(L-j-\frac{1}{2}\right), \quad j=0, \ldots, L-1, \\
\sum_{j=0}^{L-1}\left\|a^{(j)}\right\|_{C^{0}\left(I, H^{\bar{s}_{j}^{2}}(\Omega)\right)} \leq c\|a\|,
\end{gathered}
$$

and we have

$$
\left|a^{(L-1)} \partial^{2} u\right|_{0}^{2} \leq c\left|a^{(L-1)}\right|_{\bar{s}_{L-1}^{2}}^{2}\left|\partial^{2} u\right|_{\bar{s}-\bar{s}_{L-1}^{2}}^{2}
$$


so we can apply (3.5.15) to $\partial u$ with $\sigma_{1}=\bar{s}+1, \sigma_{2}=\bar{s}+1-\frac{\bar{s}+1}{L}$ :

$$
\begin{aligned}
|\partial u|_{\bar{s}+1-\bar{s}_{L-1}^{2}}^{2} & =|\partial u|_{\left(\sigma_{1}+\sigma_{2}\right) / 2}^{2} \\
& \leq c\left(\lambda^{-1}|\partial u|_{\bar{s}+1}^{2}+\lambda|\partial u|_{\bar{s}+1-(\bar{s}+1) / L}^{2}\right) \\
& \leq c\left(\lambda^{-1}|u|_{\bar{s}_{0}+2}^{2}+\lambda|u|_{\bar{s}_{1}+2}^{2}\right) \leq c\left(\lambda^{-1}|u|_{s_{0}+2}^{2}+\lambda|u|_{s_{1}+2}^{2}\right) .
\end{aligned}
$$

As a consequence we get the following estimate for $G_{7}^{t}$

$$
\begin{aligned}
G_{7}^{t} & \leq c\|a\|^{2}\left(G_{8}^{t}+G_{9}^{t}\right), \\
G_{m}^{t} & =\lambda \sum_{\substack{0 \leq l<k \leq L-1 \\
1 \leq i \leq k-l}} \varepsilon_{l}^{k} C_{l}^{k} g_{m, k, l, i}^{t}, \quad m=8,9, \\
g_{8, k, l, i}^{t} & =\lambda^{2 l-1}\left|u^{(k-l-i)}\right|_{s_{k-i}+2, \lambda, t}^{2}, \\
g_{9, k, l, i}^{t} & =\lambda^{2 l+1}\left|u^{(k-l-i)}\right|_{s_{k-i+1}+2, \lambda, t}^{2} .
\end{aligned}
$$

It is not difficult to see that if $\lambda$ is sufficiently. large so as to satisfy

$$
c\|a\|^{2} \leq 8^{-1} \lambda \min \left\{\varepsilon_{l}^{k} C_{l}^{k}: 0 \leq l<k \leq L-1\right\},
$$

then

$$
G_{7}^{t} \leq 4^{-1} G_{1}^{t}
$$

Combining (3.5.4), (3.5.6), (3.5.9), (3.5.11) and (3.5.19) we see that

$$
2^{-1} G_{1}^{t} \leq G_{5}^{t}+2^{-1} \lambda E_{2}^{t}
$$

Formulae (3.4.21) and (3.5.20) give

$$
\begin{aligned}
& 4^{-1} E_{1}^{t}+2^{-1} \lambda E_{2}^{t}+2^{-1} G_{1}^{t} \\
& \quad \leq C_{1}(\delta)\left\{E_{3}^{t}+E_{7}^{t}+G_{5}^{t}\right\}+C_{2}(\delta)\|a\|^{2} E_{8}^{t} .
\end{aligned}
$$

If $\lambda$ is sufficiently large so as to satisfy

$$
C_{2}(\delta)\|a\|^{2} \leq 4^{-1} \lambda \min \left\{\varepsilon_{l}^{k}: 0 \leq l \leq k \leq L-1\right\},
$$

then the last term in (3.5.21) can be estimated by $4^{-1} G_{1}^{t}$ and we obtain

$$
4^{-1} E_{1}^{t}+2^{-1} \lambda E_{2}^{t}+4^{-1} G_{1}^{t} \leq C(\delta)\left\{E_{3}^{t}+E_{7}^{t}+G_{5}^{t}\right\} .
$$

The proof of Theorem 3.3.1 will be complete if we prove the estimate (6.2.1). The corresponding argument is postponed to Section 6.2. 


\section{Existence theorems for non-smooth Cauchy-Dirichlet problem}

\subsection{Introductory remarks}

The local, smooth solutions to mixed problems for nonlinear, second order hyperbolic equations and systems were investigated for example in $[1,3-4,8,15$, 17-18, 22-23, 30-31, 34]. In the mentioned papers all second derivatives of solutions must be continuous on $\Omega \times I$, where $\Omega$ denotes some domain in $R^{n}$ and $I$ is a time interval. Since the initially smooth solutions may develop singularities in finite time (cf. [27], Sect. 10, and the references given there) the question of the existence of less smooth solutions arises naturally.

In the present section we consider the local solutions to the mixed, CauchyDirichlet problem for the quasilinear, hyperbolic system of the second order, without assuming that they are of class $C^{2}(\Omega \times I)$. Instead we assume that some second derivatives of solutions belong to the space $L^{2}\left(I, H^{s}(\Omega)\right), s>\frac{n}{2}$. The basic idea of the proof is similar to that presented in [8] but in our approach, the auxiliary theorems concerning the linear problem used in [8] are replaced by corresponding results from previous sections.

\subsection{Existence theorem for the linear problem}

Let us consider the linear, Cauchy-Dirichlet problem (3.2.1) with all assumptions formulated in Section 3.2. Let us assume that $\lambda \geq 0$ is a constant, $t \in I$ and recall that in (3.3.1) we have defined the basic norms used in this article. Let us also recall that the symbols $L, s_{k}, \bar{s}$ and $\delta$ were introduced in (3.2.3) and (2.2.12) respectively and define

$$
\begin{aligned}
\|a\|_{T}^{2}= & \max _{t \in(0, T)}\left|a^{(j)}(t)\right|_{\bar{s}_{j}^{1}+1}^{2}+\operatorname{ess~sup}_{t \in(0, T)}\left(\sum_{j=1}^{L-2}\left|a^{(j)}(t)\right|_{\bar{s}_{j}^{1}+1}^{2}+\left|a^{(L-1)}(t)\right|_{\bar{s}_{L-1}^{2}}^{2}\right) \\
& +\int_{0}^{T}\left\{\sum_{j=0}^{L-2}\left|a^{(j)}(t)\right|_{\bar{s}_{j}+1}^{2}+\left|a^{(L-1)}(t)\right|_{\bar{s}_{L-2}}^{2}+\left|a^{(L)}(t)\right|_{0}^{2}\right\} d t \\
\bar{s}_{j}= & \frac{\bar{s}}{L-1}(L-1-j), \quad \bar{s}_{j}^{1}=\frac{\bar{s}}{L-1}\left(L-1-j-\frac{1}{2}\right), \\
\bar{s}_{j}^{2}= & \frac{\bar{s}+1}{L}\left(L-j-\frac{1}{2}\right) .
\end{aligned}
$$

The energy inequality we have proved in Section 3 has the following consequence.

THEOREM 4.2.1. If the assumptions (2.2.6), (2.2.8), (2.2.12), (3.2.3), (3.2.4) are satisfied then there exist positive constants $C=C(\delta)$ and $\Lambda=\Lambda\left(\delta,\|a\|_{T}\right)$, such 
that the inequality

$$
\begin{array}{r}
\sum_{0 \leq j \leq L}\left(\left\|u^{(L-j)}\right\|_{0,1,0, t}^{2}+\left|u^{(L-j)}\right|_{0,1,0, t}^{2}\right)+\sum_{0 \leq l \leq k \leq L-1}\left|u^{(k-l)}\right|_{s_{k}+2,0, t}^{2} \\
\leq C(\delta) \Lambda^{2 L}\left(\delta,\|a\|_{T}\right) e^{2 \Lambda\left(\delta,\|a\|_{T}\right) T}\left\{\sum _ { 0 \leq j \leq L } \left(\left\|u^{L-j}\right\|_{0,1}^{2}\right.\right. \\
\left.\left.+\left(\int_{0}^{t}\left|f^{(L-j)}(\tau)\right|_{0} d \tau\right)^{2}\right)+\sum_{0 \leq l \leq k \leq L-1}\left|f^{(k-l)}\right|_{s_{k}, 0, t}^{2}\right\}
\end{array}
$$

holds true for arbitrary $u$ satisfying (3.2.1) and the relations

$$
\begin{aligned}
u^{(j+1)} & \in L^{\infty}\left(I, H^{0}(\Omega)\right), \\
u^{(j)} & \in L^{\infty}\left(I, H_{0}^{1}(\Omega)\right), \\
u^{(j)} & \in L^{2}\left(I, H^{s_{j}+2}(\Omega) \cap H_{0}^{1}(\Omega)\right), \quad j=0, \ldots, L-1 .
\end{aligned}
$$

Now we shall prove the following corollary from (4.2.2).

TheOREM 4.2.2. If the assumptions (2.2.6), (2,2,8), (2.2.12), (3.2.3), (3.2.4) are satisfied then there exist unique solution of the problem (3.2.1) satisfying the conditions

$$
\begin{aligned}
& u^{(j)} \in C^{0}\left(I, H^{0}(\Omega)\right), \quad j=0, \ldots, L+1, \\
& u^{(j)} \in C^{0}\left(I, H_{0}^{1}(\Omega)\right), \quad j=0, \ldots, L \\
& u^{(j)} \in L^{2}\left(I, H^{s_{j}+2}(\Omega) \cap H_{0}^{1}(\Omega)\right), \quad j=0, \ldots, L-1 .
\end{aligned}
$$

Proof. Using the regularization procedure described in Section 2 we obtain the family of smooth problems

$$
\begin{array}{ll}
u_{\alpha}^{(2)}=a_{\alpha} \partial^{2} u_{\alpha}+f_{\alpha}, & \text { in } \Omega \times I, \\
u_{\alpha}=0, & \text { on } \partial \Omega \times I, \\
u_{\alpha}(0)=u_{\alpha}^{0}, \quad u_{\alpha}^{(1)}(0)=u_{\alpha}^{1}, & \text { on } \Omega,
\end{array}
$$

with $\alpha \in(0,1)$. By the results of $[8,15]$, for $\alpha \in(0,1)$ the problem (4.2.5) possesses a solution with the regularity

$$
\begin{array}{r}
u_{\alpha}^{(j)} \in C^{0}\left(I, H^{L+1-j}(\Omega)\right), \quad j=0, \ldots, L+1, \\
u_{\alpha}^{(j)}(t) \in H_{0}^{1}(\Omega), \quad j=0, \ldots, L, \quad t \in I .
\end{array}
$$


The inequality (4.2.2) shows that the family $\left\{u_{\alpha}\right\}, \alpha \in(0,1)$ is bounded in the norm from the left hand side of (4.2.2). Consequently, we may extract a sequence $\left(u_{\mu}\right)$ such that

$$
\begin{aligned}
& u_{\mu}^{(j)} \rightarrow u^{(j)} \quad \text { weakly in } L^{2}\left(I, H^{s_{j}+2}(\Omega) \cap H_{0}^{1}(\Omega)\right), \\
& j=0, \ldots, L-1 \text {, } \\
& u_{\mu}^{(j)} \rightarrow u^{(j)} \quad \star \text { weakly in } L^{\infty}\left(I, H_{0}^{1}(\Omega)\right), \\
& j=0, \ldots, L, \\
& u_{\mu}^{(j)} \rightarrow u^{(j)} \star \text { weakly in } L^{\infty}\left(I, H^{0}(\Omega)\right), \\
& j=0, \ldots, L+1 \text {. }
\end{aligned}
$$

By the compactness lemma, cf. [19], Sect. 5.2, Chapt. 1, for some subsequence $\left(u_{\nu}\right)$ and some $n / 2<r<s$ we have

$$
u_{\nu} \rightarrow u \text { strongly in } L^{2}\left(I, H^{r+2}(\Omega)\right)
$$

Since $a_{\nu} \partial^{2} u_{\nu}-a \partial^{2} u=\left(a_{\nu}-a\right) \partial^{2} u_{\nu}+a\left(\partial^{2} u_{\nu}-\partial^{2} u\right)$, the limit function $u$ satisfies the first two conditions (3.2.1). Choose a sequence $\sigma_{k} \rightarrow 0$ and consider the mollified problems corresponding to $\sigma_{k}$ and $\sigma_{m}$. Using the notations from Sect. 5.5 and replacing $v$ by $\partial^{2} u, u_{\sigma_{k}}$ by $u_{k}, f_{\sigma_{k}}$ by $f_{k}, I_{\sigma_{k}}$ by $I_{k}$ and similarly for $m$ we obtain

$$
\begin{array}{cl}
u_{k}^{(2)}=a \partial^{2} u_{k}+f_{k}+I_{k}, & \text { in } \Omega \times I, \\
u_{k}=0, & \text { on } \partial \Omega \times I, \\
u_{m}^{(2)}=a \partial^{2} u_{m}+f_{m}+I_{m}, & \text { in } \Omega \times I, \\
u_{m}=0, & \text { on } \partial \Omega \times I,
\end{array}
$$

The difference $u_{k}-u_{m}$ satisfies

$$
\begin{array}{cl}
\left(u_{k}-u_{m}\right)^{(2)}=a \partial^{2}\left(u_{k}-u_{m}\right)+f_{k}-f_{m}+I_{k}-I_{m}, & \text { in } \Omega \times I, \\
u_{k}-u_{m}=0, & \text { on } \partial \Omega \times I .
\end{array}
$$

Repeating the argument from Section 3.4 we obtain the inequality

$$
\begin{aligned}
\mid\left(u_{k}\right. & \left.-u_{m}\right)\left.^{(L-j)}(t)\right|_{0,1, \lambda} ^{2}+2 \lambda\left|\left(u_{k}-u_{m}\right)^{(L-j)}\right|_{0,1, \lambda, t}^{2} \\
\leq & C_{j}(\delta)\left\{\left|u_{k}^{(L-j)}(0)-u_{m}^{(L-j)}(0)\right|_{0,1,0}^{2}+2\left(\left(f_{k}-f_{m}\right)^{(L-j)}\right.\right. \\
& \left.\quad+F_{1, k, m}^{L-j}+\left(I_{k}-I_{m}\right)^{(L-j)},\left(u_{k}-u_{m}\right)^{(L-j+1)}\right)_{0, \lambda, t} \\
& \left.\quad+F_{2, t, k, m}^{L-j}\left(\left(u_{k}-u_{m}\right)^{(L-j)},\left(u_{k}-u_{m}\right)^{(L-j)}\right)\right\}, \quad 0 \leq j \leq L
\end{aligned}
$$


where

$$
\begin{aligned}
& F_{1, k, m}^{L-j}= \sum_{i=1}^{L-j}\left(\begin{array}{c}
L-j \\
i
\end{array}\right) a^{(i)} \partial^{2}\left(u_{k}-u_{m}\right)^{(L-j-i)} \\
& 0 \leq j \leq L-1, \quad F_{1, k, m}^{0}=0 \\
& F_{2, t, k, m}^{j}\left(u^{(j)}, u^{(j)}\right)= \sum_{p, q=1}^{n}\left(a_{p, q}^{(1)} \partial_{q} u^{(j)}, \partial_{p} u^{(j)}\right)_{0, \lambda, t}+\gamma\left(u^{(j)}, u^{(j+1)}\right)_{0, \lambda, t} \\
&+\sum_{p, q=1}^{n}\left(\left(\partial_{p} a_{p, q}\right) \partial_{q} u^{(j)}, u^{(j+1)}\right)_{0, \lambda, t} \\
&+\sum_{\substack{p=n+1 \\
\text { or } q=n+1}}\left(a_{p, q} \partial_{p} \partial_{q} u^{(j)}, u^{(j+1)}\right)_{0, \lambda, t} .
\end{aligned}
$$

The inequalities (3.4.16), (3.4.18) and the Lemmas 5.5.1, 5.5.2 show that the right hand side in (4.2.12) tends to zero if $k, m \rightarrow \infty$. Since the constant $\lambda$ remains bounded as $k, m \rightarrow \infty$ we see that $u_{k}^{(j)}-u_{m}^{(j)} \rightarrow 0$ as $k, m \rightarrow \infty$ in the complete norm $\|\cdot\|_{0,1,0, T}$ and therefore there exists a limit function $u$ of the sequence $u_{k}$ with the properties (4.2.4). The uniqueness is also a consequence of (4.2.2).

\subsection{Existence theorem for the quasi-linear problem}

Now let us consider the quasilinear, initial-boundary value problem

$$
\begin{array}{ll}
u^{(2)}=a(\partial u) \partial^{2} u+f, & \text { in } \Omega \times I, \\
u=0, & \text { on } \partial \Omega \times I, \\
u(0)=u^{0}, \quad u^{(1)}(0)=u^{1}, & \text { on } \Omega,
\end{array}
$$

where we use the same notations as in Section 2.2 and where

$$
a(\partial u) \partial^{2} u=\sum_{p, q=1}^{n+1} a_{p, q}(\partial u) \partial_{p} \partial_{q} u
$$

We shall assume that $(2.2 .6),(3.2 .3),(3.2 .4)$ are valid and the matrix functions $a(\cdot)=\left\{a_{p, q}(\cdot): p, q=1, \ldots, n+1\right\}$, defined in some neighbourhood $U_{0}$ of the range of $\partial u^{0}$ are arbitrarily smooth for all possible $p, q$ and symmetric if $p, q \neq n+1$. Let us define the function $a^{(i)}(0)$ as the value of the derivative $\partial_{t}^{i}\{a(\partial u)\}$ at $t=0$. Using Lemma 5.4.4 one can show that the recursive definition (2.2.10) makes sense for such $a^{(i)}(0)$ and therefore the functions $u^{2}, \ldots, u^{L+1}$ are well defined provided (3.2.4) is satisfied, (cf. the argument leading to the relation (4.3.9), below). We shall assume that $u^{j}, j=0, \ldots, L+1$ satisfy (3.2.4) and additionally

$$
u^{j} \in H^{s_{j}^{2}}(\Omega), \quad s_{j}^{2}=\frac{s+2}{L+2}\left(L+2-j-\frac{1}{2}\right), \quad j=0, \ldots, L+1 .
$$


In the quasilinear case the ellipticity assumption may be formulated as follows:

$$
\delta I_{m} \sum_{p=1}^{n}\left(\xi^{p}\right)^{2} \leq \sum_{p, q=1}^{n} a_{p, q}(U) \xi^{p} \xi^{q} \leq \delta^{-1} I_{m} \sum_{p=1}^{n}\left(\xi^{p}\right)^{2}
$$

for some constant $\delta>0$ for arbitrary $\xi=\left(\xi^{1}, \ldots, \xi^{n}\right) \in R^{n}$ and arbitrary $U \in U_{0}$.

THEOREM 4.3.1. If the assumptions (2.2.6), (3.2.3), (3.2.4) with the modifications described above and (4.3.3), (4.3.4) are satisfied then there exists unique, local solution of the problem (4.3.1) with the regularity

$$
\begin{gathered}
u^{(L+1)} \in L^{\infty}\left(I^{\prime}, H^{0}(\Omega)\right), \quad u^{(L)} \in L^{\infty}\left(I^{\prime}, H_{0}^{1}(\Omega)\right), \\
u^{(j)} \in L^{2}\left(I^{\prime}, H^{s_{j}+2}(\Omega) \cap H_{0}^{1}(\Omega)\right), \\
j=0, \ldots, L-1, \quad I^{\prime}=\left(0, T^{\prime}\right) .
\end{gathered}
$$

Proof. We adopt the method of the proof from [8]. For $M, T>0$, define $Z(M, T)$ as the set of functions $w$ satisfying the following conditions

$$
\begin{aligned}
& w^{(L+1)} \in L^{\infty}\left(I, H^{0}(\Omega)\right), \quad w^{(L)} \in L^{\infty}\left(I, H_{0}^{1}(\Omega)\right), \\
& w^{(j)} \in L^{2}\left(I, H^{s_{j}+2}(\Omega) \cap H_{0}^{1}(\Omega)\right), \\
& j=0, \ldots, L-1, \quad I=(0, T), \\
& w^{(k)}(0)=u^{k}, \quad k=0, \ldots, L+1, \\
& \underset{t \in(0, T)}{\operatorname{ess} \sup }\left(\left|w^{(L+1)}(t)\right|_{0}^{2}+\left|w^{(L)}(t)\right|_{1}^{2}\right)+\int_{0}^{T}\left(\left|w^{(L-1)}\right|_{2}^{2}+|w|_{s+2}^{2}\right) d \tau \leq M^{2} \text {. }
\end{aligned}
$$

The trace theory, cf. Sect. 5.2, implies that $Z(M, T)$ is nonempty for $M$ sufficiently large. If $w \in Z(M, T)$, then one can consider the linear problem

$$
\begin{array}{ll}
u^{(2)}=a(\partial w) \partial^{2} u+f, & \text { in } \Omega \times I, \\
u=0, & \text { on } \partial \Omega \times I, \\
u(0)=u^{0}, \quad u^{(1)}(0)=u^{1}, & \text { on } \Omega .
\end{array}
$$

Using Lemmas 5.4.6, 5.4.8 and 5.4.9 one can check that the functions

$$
\begin{gathered}
a^{(L)}(\partial w) \in L^{\infty}\left(I, H^{0}(\Omega)\right), \quad a^{(L-1)}(\partial w) \in L^{2}\left(I, H^{1}(\Omega)\right), \\
a(\partial w) \in L^{2}\left(I, H^{s+1}(\Omega)\right)
\end{gathered}
$$

are bounded uniformly with respect to $w$ in corresponding spaces. It is clear that $\partial w^{(j)}(0)=\partial u^{j} \in H^{r_{j}+1}(\Omega), j=0, \ldots, L-1$ and $\partial_{t}^{j}\{a(\partial w)\}(0)$ is equal to the sum of expressions of the form $b_{l_{1} \cdots l_{k}}(\partial w(0)) \partial w^{\left(l_{1}\right)}(0) \cdots \partial w^{\left(l_{k}\right)}(0)$ for some smooth 
functions $b_{l_{1} \cdots l_{k}}$ and for $l_{1}+\cdots+l_{k}=j, 1 \leq k \leq j$. Using Lemmas 5.4.4, 5.4.5 we can check that

$$
\partial_{t}^{j}\{a(\partial w)\}(0) \in H^{r_{j}+1}(\Omega), \quad j=0, \ldots, L-1 .
$$

By the assumptions of the Theorem 4.3.1 the data in (4.3.7) satisfy the remaining assumptions of the Theorem 4.2.2, so the problem (4.3.7) is uniquely solvable on the interval $I=(0, T)$. Let us denote by $\mathcal{T}$ the map which carries $w \in Z(M, T)$ into the solution $u$ of the problem (4.3.7). We shall show that if $M$ and $T$ are appropriately chosen then $\mathcal{T}$ maps $Z(M, T)$ into itself. For this purpose we shall use Theorem 4.2.1. Choose $M_{0}>0$ such that

$$
\begin{aligned}
& M_{0}^{2} \geq 4 N_{1} C(\delta) \Lambda^{2 L}\left(\delta,\|a(\partial w)(0)\|_{0}\right), \\
& \|a(\partial w)(0)\|_{0}^{2}=\sum_{j=0}^{L-2}\left|a^{(j)}(\partial w)(0)\right|_{\bar{s}_{j}^{1}+1}^{2}, \\
& N_{T}=\sum_{0 \leq j \leq L}\left\{\left\|u^{L-j}\right\|_{0,1}^{2}+\left(\int_{0}^{T}\left|f^{(L-j)}(\tau)\right|_{0} d \tau\right)^{2}\right\} \\
& \quad+\sum_{0 \leq l \leq k \leq L-1}\left|f^{(k-l)}\right|_{s_{k}, 0, T}^{2} .
\end{aligned}
$$

The boundedness of the coefficients in the norms of the spaces listed in (4.3.8) combined with the relations

$$
\begin{aligned}
& \max _{t \in[0, T]}|v(t)-v(0)|_{0} \leq T \max _{t \in[0, T]}\left|v^{(1)}(t)\right|_{0}, \\
& |v(t)-v(0)|_{\beta} \leq c|v(t)-v(0)|_{0}^{1-\beta / m}|v(t)-v(0)|_{m}^{\beta / m}, \\
& \beta \in(0, m), \\
& \int_{0}^{T}|v(t)|_{r}^{2} d t \leq T \underset{t \in(0, T)}{\operatorname{ess} \sup _{1}|v(t)|_{r}^{2},}
\end{aligned}
$$

imply that $\left.\|a(\partial w)\|_{T^{\prime}} \rightarrow \| a(\partial w)(0)\right) \|_{0}$ as $T^{\prime} \rightarrow 0$. One can assume that $\Lambda$ depends continuousely on $\|a(\partial w)\|_{T}$ and $\|a(\partial w)\|_{T}$ depends continuously on $w \in Z(M, T)$, so if $T^{\prime} \in(0,1]$ is sufficiently small then

$$
\begin{aligned}
e^{2 \Lambda\left(\delta,\|a(\partial w)\|_{T^{\prime}}\right) T^{\prime}} & \leq 2 \\
C(\delta) N_{T^{\prime}} \Lambda^{2 L}\left(\delta,\|a(\partial w)\|_{T^{\prime}}\right) & \leq 2 C(\delta) N_{1} \Lambda^{2 L}\left(\delta,\|a(\partial w)(0)\|_{0}\right) .
\end{aligned}
$$

The relations (4.2.2), (4.3.10)-(4.3.12) show that $\mathcal{T}$ maps $Z\left(M_{0}, T^{\prime}\right)$ into itself for all sufficiently small $T^{\prime}$. 
It is sufficient to check that $\mathcal{T}$ is contractive for sufficiently small $T^{\prime}$ with respect to some complete norm. To this end for $w, \bar{w} \in Z\left(M_{0}, T^{\prime}\right)$ let us put $u=\mathcal{T} w, \bar{u}=\mathcal{T} \bar{w}, U=u-\bar{u}, W=w-\bar{w}$. The function $U$ is a solution of the problem

$$
\begin{array}{cl}
U^{(2)}=a(\partial w) \partial^{2} U+[a(\partial \bar{w})-a(\partial w)] \partial^{2} \bar{u}, & \text { in } \Omega \times\left(0, T^{\prime}\right), \\
U=0, & \text { on } \partial \Omega \times\left(0, T^{\prime}\right), \\
U(0)=0, \quad U^{(1)}(0)=0, & \text { on } \Omega .
\end{array}
$$

Repeating some arguments from Section 3.4 and using the same notations we obtain for $t \in\left(0, T^{\prime}\right)$ and $\Lambda=\Lambda\left(\delta,\|a(\partial w)\|_{T^{\prime}}\right)$

$$
\begin{aligned}
& \left|U^{(1)}(t)\right|_{0,1, \Lambda}^{2}+2 \Lambda\left|U^{(1)}\right|_{0, \Lambda, t}^{2}+a_{\gamma}(\partial U(t), \partial U(t)) \\
& +2 \Lambda \int_{0}^{t} a_{\gamma}(\partial U(\tau), \partial U(\tau)) d \tau \\
& \quad=2\left([a(\partial \bar{w})-a(\partial w)] \partial^{2} \bar{u}, U^{(1)}\right)_{0, \Lambda, t}+F_{2, t}^{0}(U, U)
\end{aligned}
$$

As in Sect. 3.4 one can prove (cf. formula (3.4.21)) that for $\Lambda$ equal to $\Lambda\left(\delta,\|a(\partial w)\|_{T^{\prime}}\right)$

$$
\begin{aligned}
\frac{1}{4} e^{-2 \Lambda T^{\prime}}\|U\|_{0,1,0, T^{\prime}}^{2} & \leq \frac{1}{4}\|U\|_{0,1, \lambda, T^{\prime}}^{2}+\lambda|U|_{0,1, \lambda, T^{\prime}}^{2} \\
& \leq C\left(\delta, M_{0}\right)\left(\int_{0}^{T^{\prime}}|a(\partial \bar{w})-a(\partial w)|_{0}\left|\partial^{2} \bar{u}\right|_{\bar{s}} d \tau\right)^{2} \\
& \leq C\left(\delta, M_{0}\right)\left(\int_{0}^{T^{\prime}}|a(\partial \bar{w})-a(\partial w)|_{0}^{2} d \tau\right)\left(\int_{0}^{T^{\prime}}\left|\partial^{2} \bar{u}\right|_{\bar{s}}^{2} d \tau\right)
\end{aligned}
$$

Applying the mean-value theorem and using the fact that $u, \bar{u}, w, \bar{w} \in Z\left(M_{0}, T^{\prime}\right)$ we get easily

$$
\begin{aligned}
& \|U\|_{0,1,0, T^{\prime}}^{2} \leq C\left(\delta, M_{0}\right) e^{2 \Lambda\left(\delta,\|a(\partial w)\|_{T^{\prime}}\right) T^{\prime}} \\
& \quad \times\left(\int_{0}^{T^{\prime}} \int_{0}^{1}|d a((1-\theta) \partial \bar{w}+\theta \partial w)|_{\bar{s}}^{2} d \theta d \tau\right)\left(\int_{0}^{T^{\prime}}\left|\partial^{2} \bar{u}\right|_{\bar{s}}^{2} d \tau\right)\|W\|_{0,1,0, T^{\prime}}^{2} \\
& \quad \leq T^{\prime} C\left(\delta, M_{0}\right) e^{2 \Lambda\left(\delta,\|a(\partial w)\|_{T^{\prime}}\right) T^{\prime}} \\
& \quad \times \max _{\tau \in\left[0, T^{\prime}\right]} \int_{0}^{1}|d a((1-\theta) \partial \bar{w}+\theta \partial w)|_{\bar{s}}^{2} d \theta\left(\int_{0}^{T^{\prime}}\left|\partial^{2} \bar{u}\right|_{\bar{s}}^{2} d \tau\right)\|W\|_{0,1,0, T^{\prime}}^{2}
\end{aligned}
$$

Since the functions in (4.3.8) are uniformly bounded in corresponding norms, from (4.3.16) it follows that the map $\mathcal{T}$ is contractive for sufficiently small $T^{\prime}$ with respect 
to the norm $\|\cdot\|_{0,1,0, T^{\prime}}$. The unique fixed point of this map gives the solution to the problem (4.3.1).

\section{Function spaces and product estimates}

\subsection{Some properties of Sobolev spaces}

In this section we collect some facts concerning the function spaces which are used in this paper. Let us begin from the remark that Theorem 2.3 of [20], Chapt. 1 has the following consequence.

Lemma 5.1.1. If $w \in L^{2}\left(I, H^{\sigma}(\Omega)\right), w^{(M)} \in L^{2}\left(I, H^{0}(\Omega)\right), \sigma \geq 0, M=$ $1,2, \ldots$, then

$$
\begin{aligned}
& w^{(j)} \in L^{2}\left(I, H^{\sigma_{j}}(\Omega)\right), \quad \sigma_{j}=\frac{\sigma}{M}(M-j), \quad j=0, \ldots, M \\
& \sum_{j=0}^{M}\left\|w^{(j)}\right\|_{L^{2}\left(I, H^{\sigma_{j}}(\Omega)\right)} \leq c\left(\|w\|_{L^{2}\left(I, H^{\sigma}(\Omega)\right)}+\left\|w^{(M)}\right\|_{L^{2}\left(I, H^{\circ}(\Omega)\right)}\right)
\end{aligned}
$$

with some positive constant $c$.

Similarly from Theorem 3.1 of [20], Chapt. 1 we can infer

LEMMA 5.1.2. If $w \in L^{2}\left(I, H^{\sigma}(\Omega)\right), w^{(M)} \in L^{2}\left(I, H^{0}(\Omega)\right), \sigma \geq 0, M=$ $1,2, \ldots$, then

$$
\begin{aligned}
& w^{(j)} \in C^{0}\left(I, H^{\sigma_{j}^{1}}(\Omega)\right), \quad \sigma_{j}^{1}=\frac{\sigma}{M}\left(M-j-\frac{1}{2}\right), \\
& j=0, \ldots, M-1, \\
& \sum_{j=0}^{M-1}\left\|w^{(j)}\right\|_{C^{0}\left(I, H^{\sigma_{j}}(\Omega)\right)} \leq c\left(\|w\|_{L^{2}\left(I, H^{\sigma}(\Omega)\right)}+\left\|w^{(M)}\right\|_{L^{2}\left(I, H^{0}(\Omega)\right)}\right),
\end{aligned}
$$

with some positive constant $c$.

\subsection{Some properties of traces}

The theory of traces from [20], Chapt. 1, Section 3.2 has the following consequences.

Lemma 5.2.1. If $f \in L^{2}\left(I, H^{\sigma}(\Omega)\right), f^{(M)} \in L^{2}\left(I, H^{0}(\Omega)\right), \sigma \geq 0, M=$ $1,2, \ldots$, then there exist traces $f^{(0)}(0), \ldots, f^{(M-1)}(0)$ of $f$ at $t=0$ satisfying the conditions

$$
\begin{gathered}
f^{(j)}(0) \in H^{\sigma_{j}^{1}}(\Omega), \quad j=0, \ldots, M-1, \quad(\text { cf. }(5.1 .2)) \\
\sum_{j=0}^{M-1}\left\|f^{(j)}(0)\right\|_{H_{j}^{\sigma_{j}^{1}(\Omega)}} \leq C\left(\|f\|_{L^{2}\left(I, H^{\sigma}(\Omega)\right)}+\left\|f^{(M)}\right\|_{L^{2}\left(I, H^{0}(\Omega)\right)}\right) \\
C=\text { const. }>0 .
\end{gathered}
$$


LEMMA 5.2.2. If $\sigma_{j}^{1}$ are as before then for every set $\left\{f^{0}, \ldots, f^{M-1}\right\}$ of functions with the properties

$$
f^{j} \in H^{\sigma_{j}^{1}}(\Omega), \quad j=0, \ldots, M-1,
$$

there exists a function $f \in L^{2}\left(I, H^{\sigma}(\Omega)\right)$ such that $f^{(M)} \in L^{2}\left(I, H^{0}(\Omega)\right)$, satisfying the relations

$$
\begin{gathered}
f^{(j)}(0)=f^{j}, \quad j=0, \ldots, M-1, \\
\|f\|_{L^{2}\left(I, H^{\sigma}(\Omega)\right)}+\left\|f^{(M)}\right\|_{L^{2}\left(I, H^{0}(\Omega)\right)} \leq C \sum_{j=0}^{M-1}\left\|f^{j}\right\|_{H_{j}^{\sigma_{j}^{1}}(\Omega)}, \\
C=\text { const. }>0 .
\end{gathered}
$$

If $f^{j} \in C^{\infty}(\bar{\Omega}), j=0, \ldots, M-1$ then one can find $f \in C^{\infty}(\bar{\Omega} \times \bar{I})$ satisfying the relation (5.2.3).

\subsection{Some product estimates}

Assume that $\Omega$ is a domain in $R^{n}$ with $C^{\infty}$ and compact boundary and let $G=\Omega$ or $G=\partial \Omega$. For $s>0, p, \theta>1, s$ non-integer, denote by $B_{p, \theta}^{s}(G)$ the Besov space of functions for which the norm defined in (5.3.5) below is finite, (the more general description of Besov spaces can be found in [35], Sect. 2.3.1, 2.5.1 and 4.2.1).

Lemma 5.3.1. Suppose $p, \theta, s_{1}, s_{2}$, are arbitrary real numbers, $s$ is real, noninteger and the following conditions are satisfied

$$
\begin{gathered}
1<p<\infty, \quad 1<\theta<\infty \\
s_{i} \geq s>0, \quad i=1,2, \quad s_{1}+s_{2}-s-\frac{n}{p}>0
\end{gathered}
$$

where $n$ denotes the dimension of $G$, then the relations

$$
u_{i} \in B_{p, \theta}^{s_{i}}(G), \quad i=1,2,
$$

imply $u_{1} u_{2} \in B_{p, \theta}^{s}(G)$ and

$$
\left\|u_{1} u_{2}\right\|_{B_{p, \theta}^{s}(G)} \leq C\left\|u_{1}\right\|_{B_{p, \theta}^{s_{1}}(G)}\left\|u_{2}\right\|_{B_{p, \theta}^{s_{2}}(G)},
$$

for some positive constant $C$. Furthermore, there exist a positive constant $C$ and small positive numbers $\varepsilon_{1}, \varepsilon_{2}$, such that $s_{i}-\varepsilon_{i}>0, i=1,2$, and for arbitrary $u_{1}$, $u_{2}$ satisfying $(5.3 .2)$

$$
\left\|u_{1} u_{2}\right\|_{B_{p, \theta}^{s}(G)} \leq C\left\|u_{1}\right\|_{B_{p, \theta}^{s_{1}-\varepsilon_{1}}(G)}\left\|u_{2}\right\|_{B_{p, \theta}^{s_{2}-\varepsilon_{2}}(G)}, \quad \text { if } s_{i}>s, \quad i=1,2
$$




$$
\begin{aligned}
& \left\|u_{1} u_{2}\right\|_{B_{p, \theta}^{s}(G)} \leq C\left(\left\|u_{1}\right\|_{B_{p, \theta}^{s_{1}-\varepsilon_{1}}(G)}\left\|u_{2}\right\|_{B_{p, \theta}^{s_{2}-\varepsilon_{2}}(G)}+\left\|u_{1}\right\|_{C^{0}(G)}\left\|u_{2}\right\|_{B_{p, \theta}^{s_{2}}(G)}\right), \\
& \text { if } s_{1}>s, \quad s_{2}=s \text {, } \\
& \left\|u_{1} u_{2}\right\|_{B_{p, \theta}^{s}(G)} \leq C\left(\left\|u_{1}\right\|_{B_{p, \theta}^{s_{1}-\varepsilon_{1}}(G)}\left\|u_{2}\right\|_{B_{p, \theta}^{s_{2}-\varepsilon_{2}}(G)}+\left\|u_{1}\right\|_{B_{p, \theta}^{s_{1}(G)}}\left\|u_{2}\right\|_{C^{0}(G)}\right), \\
& \text { if } s_{1}=s, s_{2}>s \text {, } \\
& \left\|u_{1} u_{2}\right\|_{B_{p, \theta}^{s}(G)} \leq C\left(\left\|u_{1}\right\|_{B_{p, \theta}^{s_{1}-\varepsilon_{1}}(G)}\left\|u_{2}\right\|_{B_{p, \theta}^{s_{2}-\varepsilon_{2}}(G)}\right. \\
& \left.+\left\|u_{1}\right\|_{C^{0}(G)}\left\|u_{2}\right\|_{B_{p, \theta}^{s_{2}(G)}}+\left\|u_{1}\right\|_{B_{p, \theta}^{s_{1}(G)}}\left\|u_{2}\right\|_{C^{0}(G)}\right), \\
& \text { if } s_{1}=s, \quad s_{2}=s \text {. }
\end{aligned}
$$

Proof. The case $G=\partial \Omega$ can be reduced to the case of an open domain in $R^{n-1}$ by using the local charts, cf. [36], Sect. 3.2.2. Therefore we restrict ourselves to the case of an open domain $\Omega \subset R^{n}$. In this case, $B_{p, \theta}^{s}(\Omega)$ can be characterized as the completion of the set $C^{\infty}(\Omega)$ in the norm

$$
\begin{gathered}
\|u\|_{B_{p, \theta}^{s}(\Omega)}=\|u\|_{L_{p}(\Omega)}+|u|_{B_{p, \theta}^{s}(\Omega)} \\
|u|_{B_{p, \theta}^{s}(\Omega)}=\sum_{|\alpha|=[s]}\left(\int_{R^{n}}\left\|\Delta_{h} D^{\alpha} u\right\|_{L_{p}\left(\Omega_{h}\right)}^{\theta}|h|^{-n-\{s\} \theta} d h\right)^{1 / \theta}
\end{gathered}
$$

where

$$
\begin{gathered}
{[s]=\text { the integer part of } s, \quad\{s\}=s-[s],} \\
\Omega_{h}=\left\{x \in R^{n}: x+h \in \Omega\right\}, \\
\left(\Delta_{h} \phi\right)(x)=\phi(x+h)-\phi(x), \\
D^{\alpha}=\partial_{1}^{\alpha_{1}} \cdots \partial_{n}^{\alpha_{n}}, \quad \alpha=\left(\alpha_{1}, \ldots, \alpha_{n}\right), \quad|\alpha|=\alpha_{1}+\cdots+\alpha_{n} .
\end{gathered}
$$

Since the product estimates in the $L_{p}$ norm are simple (cf. (5.3.28)) it is sufficient to estimate the terms $\left|v_{k} w_{[s]-k}\right|_{B_{p, \theta}^{\{s\}}(\Omega)}, k=0, \ldots,[s]$, where $v_{k} \in B_{p, \theta}^{s_{1}-k}(\Omega)$ is an arbitrary term of the form $D^{\alpha} u_{1},|\alpha|=k$ and $w_{[s]-k} \in B_{p, \theta}^{s_{2}-[s]+k}(\Omega)$ is an arbitrary term of the form $D^{\beta} u_{2},|\beta|=[s]-k$. According to the formula

$$
\Delta_{h}(v w)=\left(\Delta_{h} v\right)\left(\Delta_{h} w\right)+v \Delta_{h} w+\left(\Delta_{h} v\right) w
$$

we have to estimate the integrals

$$
\begin{aligned}
I_{1} & =\left(\int_{R^{n}}\left\|\left(\Delta_{h} v_{k}\right)\left(\Delta_{h} w_{[s]-k}\right)\right\|_{L_{p}\left(\Omega_{h}\right)}^{\theta}|h|^{-n-\{s\} \theta} d h\right)^{1 / \theta} \\
I_{2} & =\left(\int_{R^{n}}\left\|v_{k} \Delta_{h} w_{[s]-k}\right\|_{L_{p}\left(\Omega_{h}\right)}^{\theta}|h|^{-n-\{s\} \theta} d h\right)^{1 / \theta},
\end{aligned}
$$




$$
I_{3}=\left(\int_{R^{n}}\left\|\left(\Delta_{h} v_{k}\right) w_{[s]-k}\right\|_{L_{p}\left(\Omega_{h}\right)}^{\theta}|h|^{-n-\{s\} \theta} d h\right)^{1 / \theta} .
$$

To consider $I_{1}$ let us first note, that if $k \leq[s], r_{1}, r_{2}>1, \frac{1}{r_{1}}+\frac{1}{r_{2}}=1$, and $s_{1}, s_{2}, s$ satisfy (5.3.1), then one can choose the numbers

$$
q_{1}, q_{2}>1, \quad \frac{1}{q_{1}}+\frac{1}{q_{2}}=1, \quad \varepsilon_{1}, \varepsilon_{2}>0
$$

satisfying the conditions

$$
\begin{aligned}
s_{1}-k-\frac{\{s\}}{r_{1}}-\varepsilon_{1} & >\frac{n}{p} \frac{1}{q_{2}}, \\
s_{2}-[s]+k-\frac{\{s\}}{r_{2}}-\varepsilon_{2} & >\frac{n}{p} \frac{1}{q_{1}} .
\end{aligned}
$$

This is the consequence of the fact that the rectangle $0<x_{1}<a_{1}, 0<x_{2}<a_{2}$ and the straight line $x_{1}+x_{2}=\frac{n}{p}$ have a non-empty intersection for $a_{1}+a_{2}>\frac{n}{p}$. If $a_{1}=s_{1}-k-\frac{\{s\}}{r_{1}}-\varepsilon_{1}, a_{2}=s_{2}-[s]+k-\frac{\{s\}}{r_{2}}-\varepsilon_{2}, x_{1}=\frac{n}{p} \frac{1}{q_{2}}, x_{2}=\frac{n}{p} \frac{1}{q_{1}}$, then one can check that for sufficiently small $\varepsilon_{1}, \varepsilon_{2}>0$, the inequality $a_{1}+\varepsilon_{1} \geq s_{1}-[s]-\frac{\{s\}}{r_{1}}>$ $s_{1}-s \geq 0$, implies $a_{1}>0$ and the inequality $a_{2}+\varepsilon_{2} \geq s_{2}-[s]-\frac{\{s\}}{r_{2}}>s_{2}-s \geq 0$, implies $a_{2}>0$. It is also clear that for sufficiently small $\varepsilon_{1}, \varepsilon_{2}>0$, from the inequality $\left(a_{1}+\varepsilon_{1}\right)+\left(a_{2}+\varepsilon_{2}\right)=s_{1}+s_{2}-s>\frac{n}{p}$ it follows that $a_{1}+a_{2}>\frac{n}{p}$.

The relations (5.3.10) can be written in the form

$$
\begin{array}{r}
s_{1}-k-\varepsilon_{1}-\frac{n}{p}>\frac{\{s\}}{r_{1}}-\frac{n}{p q_{1}}, \\
s_{2}-[s]+k-\varepsilon_{2}-\frac{n}{p}>\frac{\{s\}}{r_{2}}-\frac{n}{p q_{2}},
\end{array}
$$

and (5.3.11) implies the embeddings

$$
B_{p, \theta}^{s_{1}-k-\varepsilon_{1}}(\Omega) \subset B_{p q_{1}, r_{1} \theta}^{\frac{\{s\}}{r_{1}}}(\Omega), \quad B_{p, \theta}^{s_{2}-[s]+k-\varepsilon_{2}}(\Omega) \subset B_{p q_{2}, r_{2} \theta}^{\frac{\{s\}}{r_{2}}}(\Omega)
$$

cf. [36], Theorem 3.3.1. Using Hölder's inequality and (5.3.12) we deduce that

$$
\begin{aligned}
I_{1} \leq & \left(\int\left\|\Delta_{h} v_{k}\right\|_{L_{p q_{1}}\left(\Omega_{h}\right)}^{\theta}|h|^{-\frac{1}{r_{1}}(n+\{s\} \theta)}\right. \\
& \left.\times\left\|\Delta_{h} w_{[s]-k}\right\|_{L_{p q_{2}}\left(\Omega_{h}\right)}^{\theta}|h|^{-\frac{1}{r_{2}}(n+\{s\} \theta)} d h\right)^{\frac{1}{\theta}} \\
\leq & \left(\int\left\|\Delta_{h} v_{k}\right\|_{L_{p q_{1}}\left(\Omega_{h}\right)}^{r_{1} \theta}|h|^{-(n+\{s\} \theta)} d h\right)^{\frac{1}{r_{1} \theta}}
\end{aligned}
$$




$$
\begin{aligned}
& \times\left(\int\left\|\Delta_{h} w_{[s]-k}\right\|_{L_{p q_{2}}\left(\Omega_{h}\right)}^{r_{2} \theta}|h|^{-(n+\{s\} \theta)} d h\right)^{\frac{1}{r_{2} \theta}} \\
& \leq\left\|v_{k}\right\|_{B_{p q_{1}, r_{1} \theta}^{\frac{\{s\}}{r_{1}}}}\left\|w_{[s]-k}\right\|_{B_{p q_{2}, r_{2} \theta}^{\frac{f s\}}{r_{2}}}}(\Omega) \\
& \leq C\left\|v_{k}\right\|_{B_{p, \theta}^{s_{1}-k-\varepsilon_{1}}(\Omega)}\left\|w_{[s]-k}\right\|_{B_{p, \theta}^{s_{2}-[s]+k-\varepsilon_{2}}(\Omega)} \\
& \leq C\left\|u_{1}\right\|_{B_{p, \theta}^{s_{1}-\varepsilon_{1}}(\Omega)}\left\|u_{2}\right\|_{B_{p, \theta}^{s_{2}-\varepsilon_{2}}(\Omega)} .
\end{aligned}
$$

To estimate $I_{2}$ in the case $k>0$ or $s_{2}-s>0$ we first choose the numbers (5.3.9) in such way, that

$$
s_{1}-k-\varepsilon_{1} \geq \frac{n}{p} \frac{1}{q_{2}}, \quad s_{2}-s+k-\varepsilon_{2} \geq \frac{n}{p} \frac{1}{q_{1}} .
$$

The last relations are equivalent to

$$
s_{1}-k-\varepsilon_{1}-\frac{n}{p} \geq-\frac{n}{p q_{1}}, \quad s_{2}-[s]+k-\varepsilon_{2}-\frac{n}{p} \geq\{s\}-\frac{n}{p q_{2}},
$$

and therefore the embeddings

$$
B_{p, \theta}^{s_{1}-k-\varepsilon_{1}}(\Omega) \subset L_{p q_{1}}(\Omega), \quad B_{p, \theta}^{s_{2}-[s]+k-\varepsilon_{2}}(\Omega) \subset B_{p q_{2}, \theta}^{\{s\}}(\Omega)
$$

are true. Thus in the case $k>0$ or $s_{2}-s>0$ we have

$$
\begin{aligned}
I_{2} & \leq\left(\int\left\|v_{k}\right\|_{L_{p q_{1}}\left(\Omega_{h}\right)}^{\theta}\left\|\Delta_{h} w_{[s]-k}\right\|_{L_{p q_{2}}\left(\Omega_{h}\right)}^{\theta}|h|^{-(n+\{s\} \theta)} d h\right)^{\frac{1}{\theta}} \\
& \leq\left\|v_{k}\right\|_{L_{p q_{1}}(\Omega)}\left\|w_{[s]-k}\right\|_{B_{p q_{2}, \theta}^{\{s\}}(\Omega)} \\
& \leq C\left\|v_{k}\right\|_{B_{p, \theta}^{s_{1}-k-\varepsilon_{1}}(\Omega)}\left\|w_{[s]-k}\right\|_{B_{p, \theta}^{s_{2}-[s]+k-\varepsilon_{2}}(\Omega)} \\
& \leq C\left\|u_{1}\right\|_{B_{p, \theta}^{s_{1}-\varepsilon_{1}}(\Omega)}\left\|u_{2}\right\|_{B_{p, \theta}^{s_{2}-\varepsilon_{2}}(\Omega)} .
\end{aligned}
$$

In the case $k=0$ and $s_{2}-s=0$, the last part of (5.3.1) implies $s_{1}>\frac{n}{p}$ and consequently $B_{p, \theta}^{s_{1}}(\Omega) \subset C^{0}(\Omega)$. Therefore

$$
I_{2} \leq\left\|v_{0}\right\|_{C^{0}(\Omega)}\left\|w_{[s]}\right\|_{B_{p, \theta}^{\{s\}}(\Omega)} \leq\left\|u_{1}\right\|_{C^{0}(\Omega)}\left\|u_{2}\right\|_{B_{p, \theta}^{s_{2}(\Omega)}} .
$$

The argument leading to the estimate for $I_{3}$ is analogous. If $k<[s]$ or $s_{1}>s$, one can choose the numbers (5.3.9) satisfying

$$
s_{1}-k-\{s\}-\varepsilon_{1}>\frac{n}{p} \frac{1}{q_{2}}, \quad s_{2}-[s]+k-\varepsilon_{2}>\frac{n}{p} \frac{1}{q_{1}},
$$


which imply

$$
s_{1}-k-\varepsilon_{1}-\frac{n}{p}>\{s\}-\frac{n}{p q_{1}}, \quad s_{2}-[s]+k-\varepsilon_{2}-\frac{n}{p}>-\frac{n}{p q_{2}},
$$

and consequently

$$
B_{p, \theta}^{s_{1}-k-\varepsilon_{1}}(\Omega) \subset B_{p q_{1}, \theta}^{\{s\}}(\Omega), \quad B_{p, \theta}^{s_{2}-[s]+k-\varepsilon_{2}}(\Omega) \subset L_{p q_{2}}(\Omega) .
$$

Therefore

$$
\begin{aligned}
I_{3} & \leq\left(\int\left\|\Delta_{h} v_{k}\right\|_{L_{p q_{1}}\left(\Omega_{h}\right)}^{\theta}\left\|w_{[s]-k}\right\|_{L_{p q_{2}}\left(\Omega_{h}\right)}^{\theta}|h|^{-(n+\{s\} \theta)} d h\right)^{\frac{1}{\theta}} \\
& \leq\left\|v_{k}\right\|_{B_{p q_{1}, \theta}^{\{s\}}(\Omega)}\left\|w_{[s]-k}\right\|_{L_{p q_{2}}(\Omega)} \\
& \leq C\left\|v_{k}\right\|_{B_{p, \theta}^{s_{1}-k-\varepsilon_{1}}(\Omega)}\left\|w_{[s]-k}\right\|_{B_{p, \theta}^{s_{2}-[s]+k-\varepsilon_{2}}(\Omega)} \\
& \leq C\left\|u_{1}\right\|_{B_{p, \theta}^{s_{1}-\varepsilon_{1}}(\Omega)}\left\|u_{2}\right\|_{B_{p, \theta}^{s_{2}-\varepsilon_{2}}(\Omega)}
\end{aligned}
$$

If $k=[s]$ and $s_{1}=s$ then (5.3.1) implies $s_{2}>\frac{n}{p}$, so we have $B_{p, \theta}^{s_{2}}(\Omega) \subset C^{0}(\Omega)$ and

$$
I_{3} \leq\left\|w_{0}\right\|_{C^{0}(\Omega)}\left\|v_{[s]}\right\|_{B_{p, \theta}^{\{s\}}(\Omega)} \leq\left\|u_{2}\right\|_{C^{0}(\Omega)}\left\|u_{1}\right\|_{B_{p, \theta}^{s_{1}}(\Omega)} .
$$

The proof is complete.

The first assertion of the last lemma can be formulated more simply:

$$
\begin{gathered}
B_{p, \theta}^{s_{1}}(G) B_{p, \theta}^{s_{2}}(G) \subset B_{p, \theta}^{s}(G), \quad \text { for } \quad s_{i} \geq s>0 \\
s_{1}+s_{2}-s-\frac{n}{p}>0, \quad s \text {-non-integer }
\end{gathered}
$$

Using the same convention we can formulate some simple consequences of Lemma 5.3.1 in the special case when $p, \theta=2$ and consequently $B_{p, \theta}^{s}=H^{s}$.

Lemma 5.3.2. For $L, r_{i}$ as in (2.2.3) and $0 \leq i \leq j \leq L-1$, the following embedding is true

$$
H^{r_{i}+1}(\Omega) H^{r_{j-i}}(\Omega) \subset H^{r_{j}}(\Omega)
$$

Proof. According to Lemma 5.3.1 it is sufficient to note that $r_{i}+1 \geq r_{j}$, $r_{j-i} \geq r_{j}$ and $r_{i}+1+r_{j-i}-r_{j}=s-\frac{1}{2} \frac{s}{L}+1>\frac{n}{2}$.

LEMMA 5.3.3. If the assumptions of Lemma 5.3.2 are fulfilled then

$$
H^{r_{i}+1-\frac{1}{2}}(\partial \Omega) H^{r_{j-i}+1-\frac{1}{2}}(\partial \Omega) \subset H^{r_{j}+1-\frac{1}{2}}(\partial \Omega) .
$$


Proof. The embedding (5.3.26) is a consequence of the relations $r_{i} \geq r_{j}$, $r_{j-i} \geq r_{j}$ and $r_{i}+1-1 / 2+r_{j-i}+1-1 / 2-r_{j}-1+1 / 2-(n-1) / 2=r_{i}+1+$ $r_{j-i}-r_{j}-n / 2>0$, (cf. the proof of the preceding lemma).

LEMMA 5.3.4. If $r_{j}, L$, are as before and $j=0, \ldots, L-3$, then

$$
H^{r_{j+2}+2}(\Omega) \subset H^{r_{j}}(\Omega) .
$$

Proof. It is sufficient to check that $r_{j+2}+2 \geq r_{j}, j=0, \ldots, L-3$, and one can easily show, that the last relation is equivalent to $1 \geq s / L$ and is true by assumption (2.2.3).

Let us note that in the last three lemmas we do not assume that $s$ is a noninteger. To cover the case of an integer $s$ we give also some version of Lemma 5.3.1 concerning the usual Sobolev spaces.

LEMMA 5.3.5. If $s>0$ is an integer and $s_{1}, s_{2}$, are arbitrary real numbers satisfying the conditions (5.3.1), then all the statements of Lemma 5.3 .1 remain valid, provided that the spaces $B_{p, \theta}^{s}(G)$ and $B_{p, \theta}^{s_{i}}(G)$ are replaced by the Sobolev spaces $H_{p}^{s}(G)$ and $H_{p}^{s_{i}}(G)$ respectively. If $s=0$ then the estimates (5.3.4) must be replaced by

$$
\begin{array}{lll}
\left\|u_{1} u_{2}\right\|_{L_{p}(G)} \leq C\left\|u_{1}\right\|_{H_{p}^{s_{1}-\varepsilon_{1}}(G)}\left\|u_{2}\right\|_{H_{p}^{s_{2}-\varepsilon_{2}}(G)}, & s_{1}>0, s_{2}>0 \\
\left\|u_{1} u_{2}\right\|_{L_{p}(G)} \leq\left\|u_{1}\right\|_{L_{p}(G)}\left\|u_{2}\right\|_{C^{0}(G)}, & s_{1}=0, s_{2}>0 \\
\left\|u_{1} u_{2}\right\|_{L_{p}(G)} \leq\left\|u_{1}\right\|_{C^{0}(G)}\left\|u_{2}\right\|_{L_{p}(G)}, & & s_{1}>0, s_{2}=0
\end{array}
$$

for some positive constants $C, \varepsilon_{1}, \varepsilon_{2}$ such that $s_{1}-\varepsilon_{1}>0$ and $s_{2}-\varepsilon_{2}>0$ if $s_{1}, s_{2}>0$.

Recall that if $\mathcal{S}^{\prime}\left(R^{n}\right)$ denotes the space of tempered distributions on $R^{n}$ and $\mathcal{F}$ denotes the Fourier transform on $\mathcal{S}^{\prime}\left(R^{n}\right)$ then

$$
H_{p}^{s}\left(R^{n}\right)=\left\{f \in \mathcal{S}^{\prime}\left(R^{n}\right):\|f\|_{H_{p}^{s}\left(R^{n}\right)}=\left\|\mathcal{F}^{-1}\left(1+|x|^{2}\right)^{s / 2} \mathcal{F} f\right\|_{L^{p}\left(R^{n}\right)}<\infty\right\}
$$

and $H_{p}^{s}(\Omega)$ can be defined as the space of restrictions to $\Omega$ of all functions from $H_{p}^{s}\left(R^{n}\right)$. If $s$ is a non-negative integer, then the space $H_{p}^{s}(\Omega)$ can be endowed with the norm

$$
\|f\|_{H_{p}^{s}(\Omega)}^{p}=\|f\|_{L^{p}(\Omega)}^{p}+\sum_{|\alpha|=s}\left\|D^{\alpha} f\right\|_{L^{p}(\Omega)}^{p}
$$

Proof of Lemma 5.3.5. We have to estimate the terms

$$
I=\left\|v_{k} w_{s-k}\right\|_{L_{p}(G)}, \quad k=0, \ldots, s
$$


where

$$
\begin{aligned}
v_{k} & =D^{\alpha} u_{1} \in H_{p}^{s_{1}-k}(G), \quad|\alpha|=k \\
w_{s-k} & =D^{\beta} u_{2} \in H_{p}^{s_{2}-(s-k)}(G), \quad|\beta|=s-k .
\end{aligned}
$$

In the case $s_{i}>s \geq 0, i=1,2$, one can choose the numbers (5.3.9), such that

$$
s_{1}-k-\varepsilon_{1}>\frac{n}{p} \frac{1}{q_{2}}, \quad s_{2}-s+k-\varepsilon_{2}>\frac{n}{p} \frac{1}{q_{1}},
$$

or equivalently

$$
s_{1}-k-\varepsilon_{1}-\frac{n}{p}>-\frac{n}{p q_{1}}, \quad s_{2}-s+k-\varepsilon_{2}-\frac{n}{p}>-\frac{n}{p q_{2}} .
$$

The embedding theorem, cf. [35], Sect. 4.6.1, gives

$$
\begin{aligned}
I & \leq\left\|v_{k}\right\|_{L_{p q_{1}}(G)}\left\|w_{s-k}\right\|_{L_{p q_{2}}(G)} \\
& \leq C\left\|v_{k}\right\|_{H_{p}^{s_{1}-k-\varepsilon_{1}}(G)}\left\|w_{s-k}\right\|_{H_{p}^{s_{2}-s+k-\varepsilon_{2}}(G)} \\
& \leq C\left\|u_{1}\right\|_{H_{p}^{s_{1}-\varepsilon_{1}}(G)}\left\|u_{2}\right\|_{H_{p}^{s_{2}-\varepsilon_{2}}(G)}
\end{aligned}
$$

If one of the numbers $s_{i}$ is equal to $s$, say $s_{2}=s$ and $s_{1}>s$ then $s_{1}>n / p$ and consequently $u_{1} \in C^{0}(G)$. In this case

$$
\left\|u_{1} u_{2}\right\|_{H_{p}^{s}(G)} \leq\left\|u_{1}\right\|_{C^{\circ}(G)}\left\|u_{2}\right\|_{H_{p}^{s_{2}}(G)}+\widetilde{I}
$$

where

$$
\widetilde{I}=\sum_{\substack{|\beta|<s \\|\alpha|+|\beta| \leq s}}\left\|D^{\alpha} u_{1} D^{\beta} u_{2}\right\|_{L_{p}(G)}, \quad \text { if } s>0, \quad \widetilde{I}=0, \text { if } s=0
$$

If $s=0$, then we obtain the third inequality from (5.3.28). If $s>0$, then $s_{1}-|\alpha|>0$, $s_{2}-|\beta|>0$ and $\left(s_{1}-|\alpha|\right)+\left(s_{2}-|\beta|\right) \geq s_{1}>n / p$, so the argument from the previous case, with $s$ replaced by $0, s_{1}$ replaced by $s_{1}-|\alpha|$ and $s_{2}$ replaced by $s_{2}-|\beta|$ can be applied to $\widetilde{I}$. In the case $s_{1}=s_{2}=s>0$ we have

$$
\begin{aligned}
\left\|u_{1} u_{2}\right\|_{H_{p}^{s}(G)} \leq & \left\|u_{1}\right\|_{C^{0}(G)}\left\|u_{2}\right\|_{H_{p}^{s_{2}}(G)}+\left\|u_{2}\right\|_{C^{0}(G)}\left\|u_{1}\right\|_{H_{p}^{s_{1}}(G)} \\
& +\sum_{\substack{|\alpha|<s,|\beta|<s \\
|\alpha|+|\beta| \leq s}}\left\|D^{\alpha} u_{1} D^{\beta} u_{2}\right\|_{L_{p}(G)},
\end{aligned}
$$

and the last expression can be estimated as before. 


\subsection{Some further product estimates}

In this section we shall show some further auxiliary facts used in the main text. We start from the lemma which can be obtained by using Lemma 5.3 .1 and a standard inductive argument.

Lemma 5.4.1. If $\sigma_{i} \geq s \geq 0, i=1, \ldots, l$ and for arbitrary $j_{1}<\cdots<j_{k}$, $1<k \leq l$ the inequalities $\sigma_{j_{1}}+\cdots+\sigma_{j_{k}}-(k-1) \frac{n}{2}>s$ are satisfied, then the embedding $H^{\sigma_{1}}(\Omega) \cdots H^{\sigma_{l}}(\Omega) \subset H^{s}(\Omega)$ is continuous.

Proof. It is sufficient to show that if $l>2$ and the assertion of the lemma is true for $l-1$ then it is true for $l$. In the case $\min \left\{\sigma_{1}, \ldots, \sigma_{l-1}\right\}=s$ we have for some $i<l, \sigma_{i}=s$ so $\sigma_{i}+\sigma_{l}-\frac{n}{2}>s$ implies $\sigma_{l}-\frac{n}{2}>0$. By the inductive hypothesis $H^{\sigma_{1}} \cdots H^{\sigma_{l-1}} \subset H^{s}$ and from the inequality $s+\sigma_{l}-\frac{n}{2}>s$ it follows $H^{s} H^{\sigma_{l}} \subset H^{s}$. In the case $\min \left\{\sigma_{1}, \ldots, \sigma_{l-1}\right\}>s$ let us put $\bar{s}=\min _{1 \leq k \leq l-1} \min _{i_{1}<\cdots<i_{k}}\left\{\sigma_{i_{1}}+\cdots+\right.$ $\left.\sigma_{i_{k}}-(k-1) \frac{n}{2}\right\}>\bar{s}-\varepsilon>s$, where $\varepsilon$ is a sufficiently small positive number. By the inductive hypothesis $H^{\sigma_{1}} \cdots H^{\sigma_{l-1}} \subset H^{\bar{s}-\varepsilon}$. On the other hand for some $i_{1}<\cdots<$ $i_{k}, 1 \leq k \leq l-1$, we have $\bar{s}-\varepsilon+\sigma_{l}-\frac{n}{2}=\sigma_{i_{1}}+\cdots+\sigma_{i_{k}}-(k-1) \frac{n}{2}+\sigma_{l}-\frac{n}{2}-\varepsilon>s$ so $H^{\bar{s}-\varepsilon} H^{\sigma_{l}} \subset H^{s}$.

The next three lemmas are simple consequences of Lemma 5.4.1.

LEMMA 5.4.2. If the assumption (3.2.3) is satisfied and $s_{i}^{3}=\frac{s+1}{L}\left(L-i-\frac{1}{2}\right)$, $i=0, \ldots, L-1$, then for arbitrary $j_{1}, \ldots, j_{k} \geq 1$, such that $j_{1}+\cdots+j_{k} \leq L$, the embedding $H^{s_{j_{1}}^{3}}(\Omega) \cdots H^{s_{j_{k}}^{3}}(\Omega) \subset H^{0}(\Omega)$ is continuous.

Proof. Let us observe that for $1<i \leq k, s_{j_{1}}^{3}+\cdots+s_{j_{i}}^{3}=\frac{s+1}{L}\left(i L-\left(j_{1}+\right.\right.$ $\left.\left.\cdots+j_{i}\right)-\frac{i}{2}\right)$ and therefore $s_{j_{1}}^{3}+\cdots+s_{j_{i}}^{3}-(i-1) \frac{n}{2}=(s+1)-\frac{s+1}{L}\left(j_{1}+\cdots+j_{i}\right)+$ $\left((i-1)-\frac{s+1}{L} \frac{i}{2}\right)+(i-1)\left(s-\frac{n}{2}\right)>0$.

LEMMA 5.4.3. If the assumption (3.2.3) is satisfied and $s_{i}^{3}=\frac{s+1}{L}\left(L-i-\frac{1}{2}\right)$, $s_{i}^{4}=\frac{s+1}{L}(L-i), i=1, \ldots, L-1$ then for arbitrary $j_{1}, \ldots, j_{k} \geq 1$, such that $j_{1}+\cdots+j_{k} \leq L-1$, the embedding $H^{s_{j_{1}}^{3}}(\Omega) \cdots H^{s_{j_{k-1}}^{3}}(\Omega) H^{s_{j_{k}}^{4}-1}(\Omega) \subset H^{0}(\Omega)$ is continuous.

Proof. If $\sigma_{j_{1}}=s_{j_{1}}^{3}, \ldots, \sigma_{j_{i}}=s_{j_{i}}^{3}$, then one can apply the previous argument. Assume that one of the numbers $\sigma_{j_{l}}$ is equal to $s_{j_{l}}^{4}-1$. We have for $1<i \leq k$, $\sigma_{j_{1}}+\cdots+\sigma_{j_{i}}-(i-1) \frac{n}{2}=\frac{s+1}{L}\left(i L-\left(j_{1}+\cdots+j_{i}\right)-\frac{i}{2}\right)+\frac{1}{2} \frac{s+1}{L}-1-(i-1) \frac{n}{2} \geq$ $(s+1)-\frac{s+1}{L} L+\frac{3}{2} \frac{s+1}{L}-1+\left((i-1)-\frac{s+1}{L} \frac{i}{2}\right)+(i-1)\left(s-\frac{n}{2}\right)>0$. Note that by (3.2.3) $L \geq 3$ and therefore $s+1>L-1 \geq \frac{2}{3} L$.

LEMMA 5.4.4. If the assumption (3.2.3) is satisfied and $r_{i}=\frac{s}{L}\left(L-i-\frac{1}{2}\right)$, $i=1, \ldots, L-1$, then for arbitrary $j_{1}, \ldots, j_{k} \geq 1$, such that $j_{1}+\cdots+j_{k} \leq j \leq L-1$, the embedding $H^{r_{j_{1}}+1}(\Omega) \cdots H^{r_{j_{k}}+1}(\Omega) \subset H^{r_{j}+1}(\Omega)$ is continuous. 
Proof. Similarly as before, for $1<i \leq k, r_{j_{1}}+1+\cdots+r_{j_{i}}+1=\frac{s}{L}(i L-$ $\left.\left(j_{1}+\cdots+j_{i}\right)-\frac{i}{2}\right)+i$ and therefore $r_{j_{1}}+1+\cdots+r_{j_{i}}+1-(i-1) \frac{n}{2} \geq \frac{s}{L}(L-j-$ $\left.\frac{1}{2}\right)+(i-1)\left(1-\frac{1}{2} \frac{s}{L}\right)+(i-1)\left(s-\frac{n}{2}\right)+1>r_{j}+1$.

Using the extension operator and Theorem 4 from [33] (observe that for bounded domains the assumption $b(0)=0$ is not necessary) we can prove.

Lemma 5.4.5. If $b \in C^{\infty}, G$ is an open, bounded domain in $R^{\mu}$ with the cone property and $v \in H^{\sigma}(G), \sigma>\frac{\mu}{2}$, then $b(v) \in H^{\sigma}(G)$ and $|b(v)|_{H^{\sigma}(G)} \leq$ $c\left(1+|v|_{H^{\sigma}(G)}^{\sigma}\right)$ for some constant $c>0$.

Let us note that by (3.2.3) we have $L \geq s+1>\frac{n+1}{2}$ and therefore $H^{s+1, L}(\Omega \times$ I) $\subset H^{s+1}(\Omega \times I)($ cf. $(2.2 .4))$. As a consequence we get.

LeMma 5.4.6. If $b \in C^{\infty}, \partial u \in H^{s+1, L}(\Omega \times I)$, then we have $b(\partial u) \in$ $H^{s+1}(\Omega \times I)$ and $|b(\partial u)|_{H^{s+1}(\Omega \times I)} \leq c\left(1+|\partial u|_{H^{s+1}(\Omega \times I)}^{s+1}\right)$ for some positive constant $c$.

Recall that from Lemma 5.1.2 it follows that for $\partial u \in H^{s+1, L}(\Omega \times I)$ we have $\partial u^{(j)} \in C^{0}\left(I, H^{s_{j}^{3}}(\Omega)\right), j=0, \ldots, L-1$. Combining this fact with Lemma 5.4.5 we obtain.

LEMMA 5.4.7. If $s_{0}^{3}$ is defined as in Lemma. 5.4.2 and $b \in C^{\infty}, \partial u \in$ $H^{s+1, L}(\Omega \times I)$, then we have $b(\partial u) \in C^{0}\left(I, H^{s_{0}^{3}}(\Omega)\right)$ and for some positive constant $c$,

$$
\max _{t \in I}|b(\partial u(t))|_{H_{0}^{s_{0}^{3}(\Omega)}} \leq c\left(1+\max _{t \in I}|\partial u(t)|_{H_{0}^{s_{0}^{3}(\Omega)}}^{s^{3}}\right) .
$$

Consider the derivatives with respect to $t$ of the composite function $a(\partial u)$, where $a \in C^{\infty}$. Using mollifiers one can check that the chain rule remains valid provided that the corresponding products make sense. For example $\partial_{t}^{L}\{a(\partial u)\}$ is formally equal to the sum of the term of the form $b(\partial u) \partial u^{(L)}$ and the terms of the form $b(\partial u) \partial u^{\left(j_{1}\right)} \cdots \partial u^{\left(j_{i}\right)}$, where $j_{1}+\cdots+j_{i}=L, j_{1}, \ldots, j_{i} \leq L-1$. If $\partial u^{(L)} \in L^{\infty}\left(I, H^{0}(\Omega), \partial u \in H^{s+1, L}(\Omega \times I)\right.$, then using Lemma 5.4.7 one can prove that the expression of the first kind belongs to $L^{\infty}\left(I, H^{0}(\Omega)\right)$. To prove that the expressions of the second kind belong to $L^{\infty}\left(I, H^{0}(\Omega)\right)$ it is sufficient to apply Lemmas 5.4.2, 5.4.7 and Lemma 5.1.2. Thus, we have proved the following:

Lemma 5.4.8. If $\partial u^{(L)} \in L^{\infty}\left(I, H^{0}(\Omega)\right), \partial u \in H^{s+1, L}(\Omega \times I)$ and $a(\cdot) \in C^{\infty}$ then $\partial_{t}^{L}\{a(\partial u)\} \in L^{\infty}\left(I, H^{0}(\Omega)\right)$ and the $L^{\infty}\left(I, H^{0}(\Omega)\right)$ norm of $\partial_{t}^{L}\{a(\partial u)\}$ can be estimated by some polynomial depending only on the norms $\max _{t \in I}\left|\partial u^{(0)}\right|_{s_{0}^{3}}, \ldots$, $\max _{t \in I}\left|\partial u^{(L-1)}\right|_{s_{L-1}^{3}}$, and the $L^{\infty}\left(I, H^{0}(\Omega)\right)$ norm of $\partial u^{(L)}$.

Now consider $\partial \partial_{t}^{L-1}\{a(\partial u)\}$. Using formal differentiation we can write this derivative as a sum of the expressions of the form $b(\partial u) \partial^{2} u^{(L-1)}$ and the expressions 
of the form $b(\partial u) \partial u^{\left(j_{1}\right)} \cdots \partial u^{\left(j_{i-1}\right)} \partial^{2} u^{\left(j_{i}\right)}$, where $j_{1}+\cdots+j_{i}=L-1, j_{1}, \ldots, j_{i} \leq$ $L-2$. Since $\partial^{2} u^{(L-1)} \in L^{2}\left(I, H^{0}(\Omega)\right)$ and by Lemma $5.4 .7 b(\partial u) \in C^{0}\left(I, H^{s_{0}^{3}}(\Omega)\right)$, the first kind of these expressions belongs to $L^{2}\left(I, H^{0}(\Omega)\right)$. To prove the same for the second kind of expressions it is sufficient to apply Lemma 5.4.3.

LEMMA 5.4.9. If $\partial^{2} u^{(L-1)} \in L^{2}\left(I, H^{0}(\Omega)\right), \partial u \in H^{s+1, L}(\Omega \times I)$ and $a(\cdot) \in$ $C^{\infty}$ then $\partial_{t}^{L-1}\{a(\partial u)\} \in L^{2}\left(I, H^{1}(\Omega)\right)$ and the $L^{2}\left(I, H^{1}(\Omega)\right)$ norm of $\partial_{t}^{L-1}\{a(\partial u)\}$ can be estimated by some polynomial depending only on the norms

$$
\begin{gathered}
\max _{t \in I}\left|\partial u^{(0)}\right|_{s_{0}^{3}}, \ldots, \max _{t \in I}\left|\partial u^{(L-1)}\right|_{s_{L-1}^{3}},\left\|\partial^{2} u^{(1)}\right\|_{L^{2}\left(I, H^{s_{1}^{4}-1}(\Omega)\right)}, \ldots, \\
\left\|\partial^{2} u^{(L-2)}\right\|_{L^{2}\left(I, H^{s_{L-2}^{4}}{ }^{-1}(\Omega)\right)}
\end{gathered}
$$

\subsection{Convergence of commutators}

Assume that $s, L$ are the same as in (3.2.3) and $\rho \in C_{0}^{\infty}([-2,-1])$ is such that $\int \rho(\tau) d \tau=1, \rho \geq 0$. Put $\rho_{\sigma}(\tau)=\sigma^{-1} \rho\left(\sigma^{-1} \tau\right), \sigma>0, \tau \in[0, t], v_{\sigma}(\tau)=$ $\int \rho_{\sigma}(\tau-r) v(r) d r, I_{\sigma}(\tau)=(a v)_{\sigma}(\tau)-a(\tau) v_{\sigma}(\tau)$.

LEMMA 5.5.1. If a satisfies (2.2.8) and $v \in H^{s, L-1}(\Omega \times I),(c f .(2.2 .4))$ then

$$
\int_{0}^{t}\left|I_{\sigma}^{(L)}(\tau)\right|_{0} d \tau \rightarrow 0 \quad \text { as } \sigma \rightarrow 0, \quad \text { for } t \in(0, T)
$$

Proof. Assume that $0<\sigma<\sigma_{0}$ and $\sigma_{0}$ is sufficiently small to satisfy $0<t<T-2 \sigma_{0}$. Note that for $\tau \in[0, t]$, the support of the function $r \rightarrow \rho_{\sigma}(\tau-r)$ is contained in $[\tau+\sigma, \tau+2 \sigma] \subset(0, T)$. Using the well known properties of the convolution we get

$$
\begin{aligned}
I_{\sigma}^{(L)}(\tau) & =\sum_{i=0}^{L}\left(\begin{array}{c}
L \\
i
\end{array}\right) I_{\sigma}^{i}(\tau) \\
I_{\sigma}^{i}(\tau) & =\left(a^{(i)} v^{(L-i)}\right)_{\sigma}(\tau)-a^{(i)}(\tau) v_{\sigma}^{(L-i)}(\tau)
\end{aligned}
$$

Assume first that $i>0$. Repeating the argument leading to (3.4.16), (with $j=0$ ) we get for $s_{i}, i=1, \ldots, L-1$ as in (3.2.3) and $s_{L}=-1$,

$$
\left|I_{\sigma}^{i}(\tau)\right|_{0} \leq c \int \rho_{\sigma}(\tau-r)\left|a^{(i)}(r)-a^{(i)}(\tau)\right|_{s_{i}+1}\left|v^{(L-i)}(r)\right|_{s_{L-i}} d r
$$

and by Schwarz's inequality

$$
\int_{0}^{t}\left|I_{\sigma}^{i}(\tau)\right|_{0} d \tau \leq c\left(\int_{0}^{t} \int \rho_{\sigma}(\tau-r)\left|a^{(i)}(r)-a^{(i)}(\tau)\right|_{s_{i}+1}^{2} d r d \tau\right)^{1 / 2}
$$


$(5.5 .4)$

$$
\times\left(\int_{0}^{t} \int \rho(\tau-r)\left|v^{(L-i)}(r)\right|_{s_{L-i}}^{2} d r d \tau\right)^{1 / 2} .
$$

The second integral in the right hand side of (5.5.4) is bounded since the function $w=\left|v^{(L-i)}\right|_{s_{L-i}}^{2}$ belongs to $L^{1}(I)$ and consequently $w_{\sigma}$ is bounded in $L^{1}(I)$. Using the inequality $\rho_{\sigma} \leq c \sigma^{-1}$, one can estimate the first integral by

$$
\begin{aligned}
& c \sigma^{-1} \int_{0}^{t} \int_{\tau+\sigma}^{\tau+2 \sigma}\left|a^{(i)}(r)-a^{(i)}(\tau)\right|_{s_{i}+1}^{2} d r d \tau \\
& =c \sigma^{-1} \int_{0}^{t} \int_{\sigma}^{2 \sigma}\left|a^{(i)}(\tau+\omega)-a^{(i)}(\tau)\right|_{s_{i}+1}^{2} d \omega d \tau \\
& =c \sigma^{-1} \int_{\sigma}^{2 \sigma} \int_{0}^{t}\left|a^{(i)}(\tau+\omega)-a^{(i)}(\tau)\right|_{s_{i}+1}^{2} d \tau d \omega \\
& \quad \leq c \sup _{\sigma \leq \omega \leq 2 \sigma} \int_{0}^{t}\left|a^{(i)}(\tau+\omega)-a^{(i)}(\tau)\right|_{s_{i}+1}^{2} d \tau .
\end{aligned}
$$

The last term in (5.5.5) converges to zero as $\sigma \rightarrow 0$ since $a^{(i)} \in L^{2}\left(I, H^{s_{i}+1}(\Omega)\right)$, $i=1, \ldots, L$.

Now consider

$$
\begin{aligned}
I_{\sigma}^{0} & =\left(a v^{(L)}\right)_{\sigma}-a\left(v^{(L)}\right)_{\sigma} \\
& =\partial_{\tau}\left[\left(a v^{(L-1)}\right)_{\sigma}-a\left(v^{(L-1)}\right)_{\sigma}\right]=J_{1}+J_{2}+J_{3},
\end{aligned}
$$

where

$$
\begin{aligned}
& J_{1}(\tau)=\int \partial_{r}\left(\rho_{\sigma}(\tau-r)\right)(a(\tau)-a(r))\left(v^{(L-1)}(r)-v^{(L-1)}(\tau)\right) d r \\
& J_{2}(\tau)=-\int \rho_{\sigma}(\tau-r) a^{(1)}(r)\left(v^{(L-1)}(r)-v^{(L-1)}(\tau)\right) d r \\
& J_{3}(\tau)=\int \rho_{\sigma}(\tau-r)\left(a^{(1)}(r)-a^{(1)}(\tau)\right) v^{(L-1)}(r) d r
\end{aligned}
$$

Since $s_{1}+1=s-\frac{s}{L-1}+1>\frac{n}{2}$, we have

$$
\begin{aligned}
\left|J_{1}(\tau)\right|_{0} \leq c \int \mid & \left|\partial_{r}\left(\rho_{\sigma}(\tau-r)\right)(a(\tau)-a(r))\right|_{s_{1}+1} \\
& \times\left|\left(v^{(L-1)}(r)-v^{(L-1)}(\tau)\right)\right|_{0} d r .
\end{aligned}
$$

Using the estimate for the difference quotient from Lemma 5.5.3, below and the inequality

$$
\left|\omega \partial_{\omega}\left(\rho_{\sigma}(\omega)\right)\right| \leq c \sigma^{-1}
$$


we obtain

$$
\begin{aligned}
& \int_{0}^{t} \int_{\tau+\sigma}^{\tau+2 \sigma}\left|\partial_{r}\left(\rho_{\sigma}(\tau-r)\right)\right||a(\tau)-a(r)|_{s_{1}+1}\left|v^{(L-1)}(r)-v^{(L-1)}(\tau)\right|_{0} d r d \tau \\
&=\int_{0}^{t} \int_{\tau+\sigma}^{\tau+2 \sigma}|\tau-r|\left|\partial_{r}\left(\rho_{\sigma}(\tau-r)\right)\right| \\
& \quad \times\left|(\tau-r)^{-1}(a(\tau)-a(r))\right|_{s_{1}+1}\left|v^{(L-1)}(r)-v^{(L-1)}(\tau)\right|_{0} d r d \tau \\
& \leq c \sigma^{-1} \int_{\sigma}^{2 \sigma}\left(\int_{0}^{t}\left|\omega^{-1}(a(\tau+\omega)-a(\tau))\right|_{s_{1}+1}^{2} d \tau\right)^{1 / 2} \\
& \times\left(\int_{0}^{t}\left|v^{(L-1)}(\tau+\omega)-v^{(L-1)}(\tau)\right|_{0}^{2} d \tau\right)^{1 / 2} d \omega \\
& \leq c\left(\int_{0}^{T}\left|a^{(1)}(r)\right|_{s_{1}+1}^{2} d r\right) \\
& \times \sup _{\sigma \leq \omega \leq 2 \sigma}\left(\int_{0}^{t}\left|v^{(L-1)}(\tau+\omega)-v^{(L-1)}(\tau)\right|_{0}^{2} d \tau\right)^{1 / 2} .
\end{aligned}
$$

Since $v^{(L-1)} \in L^{2}\left(I, H^{0}(\Omega)\right)$, the last term in (5.5.10), and consequently $J_{1}$ converges to zero as $\sigma \rightarrow 0$. To prove the same for $J_{3}$, it is sufficient to repeat the argument leading to (5.5.3)-(5.5.5), with $i=1$, and to consider $J_{2}$ it is sufficient to replace in this argument $\left|a^{(i)}(r)-a^{(i)}(\tau)\right|_{s_{1}+1}$ by $\left|v^{(L-1)}(r)-v^{(L-1)}(\tau)\right|_{0}$ and $\left|v^{(L-i)}(r)\right|_{s_{L-i}}$ by $\left|a^{(1)}(r)\right|_{s_{1}+1}$.

From the proof of Lemma 5.5.1 the next result follows easily.

LEMMA 5.5.2. The assertion of Lemma 5.5.1 remains valid if the number $L$ in (5.5.1) is replaced by arbitrary $j=0, \ldots, L-1$.

For completeness we also give the proof of the following result.

Lemma 5.5.3. If $t \in(0, T), 0<h_{0}<T-t$ and

$$
a, a^{(1)} \in L^{p}((0, T), X), \quad p \geq 1,
$$

where $X$ is a Banach space with the norm $\|\cdot\|_{X}$, then

$$
\sup _{0<h<h_{0}} \int_{0}^{t}\left\|h^{-1}(a(\tau+h)-a(\tau))\right\|_{X}^{p} d \tau \leq \int_{0}^{T}\left\|a^{(1)}(r)\right\|_{X}^{p} d r .
$$

Proof: Since the functions satisfying (5.5.11) are absolutely continuous with respect to $t$, we have for $0<h<h_{0}$ and $\tau \in[0, t]$

$$
h^{-1}(a(\tau+h)-a(\tau))=h^{-1} \int_{\tau}^{\tau+h} a^{(1)}(r) d r .
$$


Hölder's inequality gives

$$
\begin{aligned}
\left\|h^{-1}(a(\tau+h)-a(\tau))\right\|_{X}^{p} & =h^{-1}\left\|\int_{\tau}^{\tau+h} a^{(1)}(r) d r\right\|_{X}^{p} \\
& \leq h^{-1} \int_{\tau}^{\tau+h}\left\|a^{(1)}(r)\right\|_{X}^{p} d r
\end{aligned}
$$

Integrating over $(0, t)$ we get

$$
\begin{aligned}
\int_{0}^{t}\left\|h^{-1}(a(\tau+h)-a(\tau))\right\|_{X}^{p} d \tau & \leq h^{-1} \int_{0}^{t} \int_{\tau}^{\tau+h}\left\|a^{(1)}(r)\right\|_{X}^{p} d r d \tau \\
& =h^{-1} \int_{0}^{h} \int_{0}^{t}\left\|a^{(1)}(\tau+\omega)\right\|_{X}^{p} d \tau d \omega .
\end{aligned}
$$

Since

$$
\begin{aligned}
\int_{0}^{t}\left\|a^{(1)}(\tau+\omega)\right\|_{X}^{2} d \tau & =\int_{\omega}^{t+\omega}\left\|a^{(1)}(r)\right\|_{X}^{p} d r \\
& \leq \int_{0}^{t+h}\left\|a^{(1)}(r)\right\|_{X}^{p} d r \leq \int_{0}^{T}\left\|a^{(1)}(r)\right\|_{X}^{p} d r
\end{aligned}
$$

the proof is complete.

\section{Some elliptic estimates}

\subsection{Elliptic estimates independent of $t$}

Fix the numbers $1<p, \theta<\infty$ and denote by $B_{p, \theta}^{s}(G), s>0, G \subset R^{k}$ the Besov space, defined for example in [36], p. 192, (cf. also Sect. 5.3). In this section we shall show that there exist constants $C(\delta)$ and $C\left(\|a\|_{B_{p, \theta}^{s^{0}+1}(\Omega)}\right)$ such that for arbitrary $u \in B_{p, \theta}^{s+2}(\Omega)$ the following inequality holds true

$$
\begin{gathered}
\|u\|_{B_{p, \theta}^{s+2}(\Omega)} \leq C(\delta)\left\{\left\|a \partial^{2} u\right\|_{B_{p, \theta}^{s}(\Omega)}+\|b \partial u\|_{B_{p, \theta}^{s+1-\frac{1}{p}}(\partial \Omega)}\right. \\
\left.+C\left(\|a\|_{B_{p, \theta}^{s^{0}+1}(\Omega)}\right)\|u\|_{L_{p}(\Omega)}\right\}
\end{gathered}
$$

Here by assumption

$$
\begin{gathered}
\quad s \text { and } s+1-\frac{1}{p} \text { are non-integer and } \\
0 \leq s \leq s^{0}, \quad \text { for some } s^{0} \text { satisfying } s^{0}+1>\frac{n}{p} .
\end{gathered}
$$

If $s$ is an integer (6.1.1) remains valid with $B_{p, \theta}^{s}(\Omega)$ replaced by $H_{p}^{s}(\Omega)$ and $B_{p, \theta}^{s}(\partial \Omega)$ replaced by $B_{p, p}^{s}(\partial \Omega)$. The operators $a \partial^{2}$ and $b \partial$ are defined in (2.2.2) and $a \partial^{2}$ 
satisfies (2.2.12). The estimate (6.1.1) corresponds to the Neumann problem. In the case of the homogeneous Dirichlet problem it is sufficient to drop the boundary term in (6.1.1). It is of a fundamental importance that we consider coefficients $a=\left\{a_{p, q}\right\}$ with the regularity

$$
a \in B_{p, \theta}^{s^{0}+1}(\Omega) .
$$

We shall sketch a proof of (6.1.1) in the special case $\Omega=R_{+}^{n}$, assuming $u$ is sufficiently smooth with small support. The usual procedure of localization allows for extending our result to the general case of smooth domain with compact boundary. The general scheme of the proof is the same as in the Appendix of [15].

Assume that $u$ has small support in which the oscilations of the coefficients $a=\left\{a_{p, q}\right\}$ are small. Recall, that in the constant coefficients case the following inequality is true for sufficiently smooth $u$

$$
\|u\|_{B_{p, \theta}^{s+2}\left(R_{+}^{n}\right)} \leq C(\delta)\left\{\left\|a \partial^{2} u\right\|_{B_{p, \theta}^{s}\left(R_{+}^{n}\right)}+\|b \partial u\|_{B_{p, \theta}^{s+1-\frac{1}{p}}\left(R^{n-1}\right)}+\|u\|_{L_{p}\left(R_{+}^{n}\right)}\right\}
$$

cf. [36], Sect. 4.2.3. Therefore it is sufficient to prove the following perturbation estimate.

Lemma 6.1.1. For arbitrary $\varepsilon>0$, there exist $\rho>0$ such that if $u \in$ $B_{p, \theta}^{s+2}\left(R_{+}^{n}\right)$ is a function with support in the ball $|x|<\rho$, the real numbers $s, p$, $\theta$ satisfy (6.1.2) and $1<p, \theta<\infty$, then the following inequality holds true

$$
\begin{aligned}
\| a \partial^{2} u & -a_{0} \partial^{2} u\left\|_{B_{p, \theta}^{s}\left(R_{+}^{n}\right)}+\right\| b \partial u-b_{0} \partial u \|_{B_{p, \theta}^{s+1-\frac{1}{p}}\left(R^{n-1}\right)} \\
& \leq \varepsilon\|u\|_{B_{p, \theta}^{s+2}\left(R_{+}^{n}\right)}+C\left(\varepsilon,\|a\|_{B_{p, \theta}^{s^{0}+1}\left(R_{+}^{n}\right)}\right)\|u\|_{L_{p}\left(R_{+}^{n}\right)}
\end{aligned}
$$

where $a_{0} \partial^{2}$ and $b_{0} \partial$ are the constant coefficients operators obtained from a $\partial^{2}$ and bə by freezing the coefficients at $x=0$.

Proof. Let us assume that $C$ denotes the constant from (5.3.4) and

$$
C \sup _{|x|<\rho}|a(x)-a(0)| \leq \frac{1}{4} \varepsilon
$$

The relation (6.1.6) is satisfied for sufficiently small $\rho$ since the inequality $s^{0}+1>$ $n / p$ implies $B_{p, \theta}^{s^{0}+1}\left(R_{+}^{n}\right) \subset C^{0}\left(R_{+}^{n}\right)$, cf. [35], p. 328. From Lemma 5.3.1 it follows

$$
\begin{aligned}
J_{1} & =\left\|a \partial^{2} u-a_{0} \partial^{2} u\right\|_{B_{p, \theta}^{s}\left(R_{+}^{n}\right)} \\
& \leq C\left\|a-a_{0}\right\|_{C^{0}\left(R_{+}^{n}\right)}\|u\|_{B_{p, \theta}^{s+2}\left(R_{+}^{n}\right)}
\end{aligned}
$$




$$
\begin{aligned}
& +C\left\|a-a_{0}\right\|_{B_{p, \theta}^{s^{0}+1}\left(R_{+}^{n}\right)}\|u\|_{B_{p, \theta}^{s+2-\varepsilon_{2}}\left(R_{+}^{n}\right)} \\
\leq & \frac{1}{4} \varepsilon\|u\|_{B_{p, \theta}^{s+2}\left(R_{+}^{n}\right)} \\
& +C\left\|a-a_{0}\right\|_{B_{p, \theta}^{s^{0}+1}\left(R_{+}^{n}\right)}\left(\varepsilon_{2}\|u\|_{B_{p, \theta}^{s+2}\left(R_{+}^{n}\right)}+C_{\varepsilon_{2}}\|u\|_{L_{p}\left(R_{+}^{n}\right)}\right) .
\end{aligned}
$$

Putting $\varepsilon_{2}=\frac{1}{4} \varepsilon C^{-1}\left(1+\left\|a-a_{0}\right\|_{B_{r, \theta}^{s^{0}+1}\left(R_{+}^{n}\right)}\right)^{-1}$, we obtain

$$
J_{1} \leq \frac{1}{2} \varepsilon\|u\|_{B_{p, \theta}^{s+2}\left(R_{+}^{n}\right)}+C\left(\varepsilon,\left\|a-a_{0}\right\|_{B_{p, \theta}^{s^{0}+1}\left(R_{+}^{n}\right)}\right)\|u\|_{L_{p}\left(R_{+}^{n}\right)} .
$$

The boundary term can be estimated analogously because

$$
\begin{aligned}
J_{2}= & \left\|b \partial u-b_{0} \partial u\right\|_{B_{p, \theta}^{s+1-\frac{1}{p}}\left(R^{n-1}\right)} \| \\
\leq & C\left\|b-b_{0}\right\|_{C^{0}\left(R^{n-1}\right)}\|\partial u\|_{B_{p, \theta}^{s+1-\frac{1}{p}}\left(R^{n-1}\right)}\|\partial u\|_{B_{p, \theta}^{s+1-\frac{1}{p}-\varepsilon_{2}}\left(R^{n-1}\right)} \\
& +C\left\|b-b_{0}\right\|_{B_{p, \theta}^{s^{0}+1-\frac{1}{p}}\left(R^{n-1}\right)}\|\partial u\|_{B_{p, \theta}^{s^{0}+1}\left(R_{+}^{n}\right)}\|u\|_{B_{p, \theta}^{s+2-\varepsilon_{2}}\left(R_{+}^{n}\right)} .
\end{aligned}
$$

\subsection{Elliptic estimates with $L^{2}$ dependence on $t$}

The present section is devoted to the estimate of the form

$$
\begin{aligned}
\int_{0}^{t} e^{-2 \lambda \tau}\|u(\tau)\|_{B_{p, \theta}^{s+2}(\Omega)}^{2} d \tau \leq & C(\delta)\left\{\int_{0}^{t} e^{-2 \lambda \tau}\left\|a \partial^{2} u(\tau)\right\|_{B_{p, \theta}^{s}(\Omega)}^{2} d \tau\right. \\
& +\int_{0}^{t} e^{-2 \lambda \tau}\left\|B^{d} u(\tau)\right\|_{B_{p, \theta}^{s+d-1 / p}(\partial \Omega)}^{2} d \tau \\
& \left.+C\left(\|a\|_{C^{0}\left(I, B_{p, \theta}^{s^{0}+1}(\Omega)\right)}\right) \int_{0}^{t} e^{-2 \lambda \tau}\|u(\tau)\|_{L_{p}(\Omega)}^{2} d \tau\right\}
\end{aligned}
$$

where $u$ is an arbitrary function satisfying the inclusion $u \in L^{2}\left(I, B_{p, \theta}^{s+2}(\Omega)\right)$, and the symbols $C(\delta), C\left(\|a\|_{C^{0}\left(I, B_{p, \theta}^{s^{0}+1}(\Omega)\right)}\right)$ denote some positive constants depending on the indicated parameters. The operator $a \partial^{2}$ is the same as in (2.2.2) and $B^{d}$, $d=0,1$ denotes the Dirichlet or Neumann boundary operator

$$
\begin{aligned}
& B^{0} u=u \\
& B^{1} u=\sum_{p, q=1}^{n} \nu_{p} a_{p, q} \partial_{q} u
\end{aligned}
$$


where $\nu=\left(\nu_{1}, \ldots, \nu_{n}\right)$ is the unit, outward, normal vector to the boundary $\partial \Omega$. Recall that $\partial \Omega$ is assumed to be $C^{\infty}$ and compact. Additionally, we assume that

$$
\begin{gathered}
s, s+2-d-1 / p \quad \text { are non-integers } \\
0 \leq s \leq s^{0}, \quad \frac{n}{p}<s^{0}+1
\end{gathered}
$$

and the coefficients $a=\left\{a_{p, q}\right\}$ have the regularity

$$
a \in C^{0}\left(I, B_{p, \theta}^{s^{0}+1}(\Omega)\right)
$$

We repeat some statements from the previous section to enable the reader to control the regularity requirements concerning the coefficients. Thus, as in the previous section, we shall sketch the proof of (6.2.1) in special case $\Omega=R_{+}^{n}$, assuming $u$ is smooth, with small support in which the oscilations of the coefficients $a_{p, q}$ are small. If $a_{0}=\left\{a_{p, q}\left(x_{0}, t_{0}\right)\right\}, B_{0}^{d}=B^{d}\left(x_{0}, t_{0}\right)$, i.e. the variables of the coefficients are fixed at some $\left(x_{0}, t_{0}\right)$, then from [36], Sect. 4.2.3 we can deduce the following inequality for $u \in B_{p, \theta}^{s+2}\left(R_{+}^{n}\right)$

$$
\begin{aligned}
\|u\|_{B_{p, \theta}^{s+2}\left(R_{+}^{n}\right)}^{2} \leq C(\delta) & \left(\left\|a_{0} \partial^{2} u\right\|_{B_{p, \theta}^{s}\left(R_{+}^{n}\right)}^{2}\right. \\
& \left.+\left\|B_{0}^{d} u\right\|_{B_{p, \theta}^{s+2-d-1 / p}\left(R^{n-1}\right)}^{2}+\|u\|_{L_{p}\left(R_{+}^{n}\right)}^{2}\right) .
\end{aligned}
$$

Multiplying both sides of (6.2.5) by $e^{-2 \lambda \tau}$ and integrating over $(0, t)$ we see that

$$
\begin{aligned}
\int_{0}^{t} e^{-2 \lambda \tau}\|u(\tau)\|_{B_{p, \theta}^{s+2}\left(R_{+}^{n}\right)}^{2} d \tau \leq C(\delta)\{ & \left.\int_{0}^{t} e^{-2 \lambda \tau} \| a_{0} \partial^{2} u(\tau)\right) \|_{B_{p, \theta}^{s}\left(R_{+}^{n}\right)}^{2} d \tau \\
& +\int_{0}^{t} e^{-2 \lambda \tau}\left\|B_{0}^{d} u(\tau)\right\|_{B_{p, \theta}^{s+2-d-1 / p}\left(R^{n-1}\right)}^{2} d \tau \\
& \left.+\int_{0}^{t} e^{-2 \lambda \tau}\|u(\tau)\|_{L_{p}\left(R_{+}^{n}\right)}^{2} d \tau\right\}
\end{aligned}
$$

Inequality (6.2.6) shows that to obtain (6.2.1) for $\Omega=R_{+}^{n}$ it is sufficient to prove the pertubation estimate from the following lemma.

LEMMA 6.2.1. For all, sufficiently small $\varepsilon>0$, there exist $\rho>0$, such that if $u \in L^{2}\left(I, B_{p, \theta}^{s+2}\left(R_{+}^{n}\right)\right)$ has support in

$$
K=\left\{(x, t) \in R_{+}^{n} \times I:|x|<\rho,\left|t-t_{0}\right|<\rho\right\},
$$

then for arbitrary s, $p, \theta, \quad 1<p, \theta<\infty$ satisfying (6.2.3) and for some positive 
constant $C\left(\varepsilon,\|a\|_{C^{0}\left(I, B_{p, \theta}^{s^{0}+1}\left(R_{+}^{n}\right)\right)}\right)$,

$$
\begin{aligned}
& \int_{0}^{t} e^{-2 \lambda \tau}\left\|a \partial^{2} u(\tau)-a_{0} \partial^{2} u(\tau)\right\|_{B_{p, \theta}^{s}\left(R_{+}^{n}\right)}^{2} d \tau \\
& +\int_{0}^{t} e^{-2 \lambda \tau}\left\|B^{d} u(\tau)-B_{0}^{d} u(\tau)\right\|_{B_{p, \theta}^{s+2-d-1 / p}\left(R^{n-1}\right)}^{2} d \tau \\
& \leq \varepsilon \int_{0}^{t} e^{-2 \lambda \tau}\|u(\tau)\|_{B_{p, \theta}^{s+2}\left(R_{+}^{n}\right)}^{2} d \tau \\
& \quad+C\left(\varepsilon,\|a\|_{C^{0}\left(I, B_{p, \theta}^{s^{0}+1}\left(R_{+}^{n}\right)\right)}\right) \int_{0}^{t} e^{-2 \lambda \tau}\|u(\tau)\|_{L_{p}\left(R_{+}^{n}\right)}^{2} d \tau
\end{aligned}
$$

where $a_{0} \partial^{2}$ and $B_{0}^{d}$ denote the operators with constant coefficients obtained from $a \partial^{2}$ and $B^{d}$ respectively, by freezing the coefficients at $(x, t)=\left(0, t_{0}\right), t_{0} \in I$.

Proof. Assume that $\varepsilon$ is a small positive number, $C$ is the constant from Lemma 5.3.1 and

$$
C \sup _{(x, t) \in K}\left|a-a_{0}\right| \leq 4^{-1} \varepsilon
$$

for some $K$ as in (6.2.7). Similarly as for (6.1.6), (6.2.9) is satisfied for sufficiently small $\rho$. Using Lemma 5.3.1 we get for some $\varepsilon_{2}>0$

$$
\begin{aligned}
I_{1}= & \int_{0}^{t} e^{-2 \lambda \tau}\left\|a \partial^{2} u(\tau)-a_{0} \partial^{2} u(\tau)\right\|_{B_{p, \theta}^{s}\left(R_{+}^{n}\right)}^{2} d \tau \\
\leq & C \int_{0}^{t} e^{-2 \lambda \tau}\left\|a-a_{0}\right\|_{C^{0}(K)}\|u(\tau)\|_{B_{p, \theta}^{s+2}\left(R_{+}^{n}\right)}^{2} d \tau \\
& +C \int_{0}^{t} e^{-2 \lambda \tau}\left\|a-a_{0}\right\|_{B_{p, \theta}^{s^{0}+1}\left(R_{+}^{n}\right)}\|u(\tau)\|_{B_{p, \theta}^{s+2-\varepsilon_{2}}\left(R_{+}^{n}\right)}^{2} d \tau \\
\leq & 4^{-1} \varepsilon \int_{0}^{t} e^{-2 \lambda \tau}\|u(\tau)\|_{B_{p, \theta}^{s+2}\left(R_{+}^{n}\right)}^{2} d \tau \\
& +C\left\|a-a_{0}\right\|_{C^{0}\left(B_{p, \theta}^{s^{0}+1}\left(R_{+}^{n}\right)\right)}\left(\varepsilon_{2} \int_{0}^{t} e^{-2 \lambda \tau}\|u(\tau)\|_{B_{p, \theta}^{s+2}\left(R_{+}^{n}\right)}^{2} d \tau\right. \\
& \left.\quad+C_{\varepsilon_{2}} \int_{0}^{t} e^{-2 \lambda \tau}\|u(\tau)\|_{L_{p}\left(R_{+}^{n}\right)}^{2} d \tau\right) .
\end{aligned}
$$

For sufficiently small $\varepsilon$ we can put

$$
\varepsilon_{2}=4^{-1} \varepsilon C^{-1}\left(1+\left\|a-a_{0}\right\|_{C^{0}\left(I, B_{p, \theta}^{s^{0}+1}\left(R_{+}^{n}\right)\right)}\right)^{-1}
$$


and consequently we have

$$
\begin{aligned}
I_{1} \leq & 2^{-1} \varepsilon \int_{0}^{t} e^{-2 \lambda \tau}\|u(\tau)\|_{B_{p, \theta}^{s+2}\left(R_{+}^{n}\right)}^{2} d \tau \\
& +C\left(\varepsilon,\left\|a-a_{0}\right\|_{C^{0}\left(I, B_{p, \theta}^{s^{0}+1}\left(R_{+}^{n}\right)\right)}\right) \int_{0}^{t} e^{-2 \lambda \tau}\|u(\tau)\|_{L_{p}\left(R_{+}^{n}\right)}^{2} d \tau .
\end{aligned}
$$

In the case of the Dirichlet boundary condition, the boundary term in (6.2.8) vanishes so it is sufficient to consider the boundary term corresponding to the Neumann problem. Applying Lemma 5.3.1 with $G=\partial R_{+}^{n}=R^{n-1}$ we obtain

$$
\begin{aligned}
I_{2}= & \int_{0}^{t} e^{-2 \lambda \tau}\left\|B^{1} u(\tau)-B_{0}^{1} u(\tau)\right\|_{B_{p, \theta}^{s+1-1 / p}\left(R^{n-1}\right)}^{2} d \tau \\
\leq & C \int_{0}^{t} e^{-2 \lambda \tau}\left\|a-a_{0}\right\|_{C^{0}\left(K_{0}\right)}^{2}\|u(\tau)\|_{B_{p, \theta}^{s+2-1 / p}\left(R^{n-1}\right)}^{2} d \tau \\
& +C \int_{0}^{t} e^{-2 \lambda \tau}\left\|a-a_{0}\right\|_{B_{p, \theta}^{s^{0}+1-1 / p}\left(R^{n-1}\right)}^{2}\|u(\tau)\|_{B_{p, \theta}^{s+2-1 / p}\left(R^{n-1}\right)}^{2} d \tau
\end{aligned}
$$

where $K_{0}=K \cap\left\{x \in R^{n}: x_{n}=0\right\}$. Since arbitrary $w \in B_{p, \theta}^{r}\left(R_{+}^{n}\right), r>1 / p$, $p, \theta>1$, satisfies the inequality

$$
\|w\|_{B_{p, \theta}^{r-1 / p}\left(R^{n-1}\right)} \leq C\|w\|_{B_{p, \theta}^{r}\left(R_{+}^{n}\right)}, \quad c=\text { const }>0
$$

we can repeat the preceding argument to obtain for $I_{2}$ the same estimate as for $I_{1}$.

It is worth noting that to estimate $I_{1}$, the relation

$$
s \in\left[0, s^{0}+1\right]
$$

is sufficient while to estimate $I_{2}$ we need $(6.2 .3)$.

The standard procedure of localization and deformation extends the obtained estimate to the case of general $\Omega$.

Lemma 6.2.2. If $\Omega \subset R^{n}$ is a bounded domain with a $C^{\infty}$ and compact boundary $\partial \Omega, I \subset R^{1}$ is a finite interval and the assumptions (2.2.12), (6.2.3), (6.2.4) are fulfilled, then the estimate $(6.2 .1)$ is valid for arbitrary $u \in$ $L^{2}\left(I, B_{p, \theta}^{s+2}(\Omega)\right)$. If $u$ satisfies additionally the homogeneous Dirichlet condition, then the estimate (6.2.1) with dropped boundary term remains valid for a satisfying (6.2.14).

Using Lemma 5.3.5 one also can prove the following modified version of Lemma 6.2.2. 
Lemma 6.2.3. If $s$ is an integer then all the statements of Lemma 6.2 .2 remain valid provided that the Besov spaces $B_{p, \theta}^{s}(\Omega), B_{p, \theta}^{s+2-d-1 / p}(\partial \Omega)$ are replaced by the Sobolev space $H_{p}^{s}(\Omega)$ and the Besov space $B_{p, p}^{s+2-d-1 / p}(\partial \Omega)$ respectively.

\section{References}

[ 1 ] H. Beirao da Veiga, Structural stability and data dependence for fully non-linear hyperbolic mixed problems, Arch. Rational Mech. Anal., 120 (1992), 51-60.

[ 2 ] J. Chazarain and A. Piriou, Introduction a la théorie des équations aux dérivées partielles linéaires, Gauthier-Villars, Paris, 1981.

[ 3 ] A. Chrzęszczyk, Initial-boundary value prolems for non-linear equations of generalized thermoelasticity and elasticity, Math. Meth. in the Appl. Sci., 16 (1993), 49-60.

[ 4 ] A. Chrzęszczyk, On some class of initial boundary value problems for second order quasilinear hyperbolic systems, Tsukuba J. Math., 16 (1992), 443-486.

[ 5 ] A. Chrzęszczyk, On some second-order non-linear systems of a hyperbolic-parabolic type, Math. Meth. in the Appl. Sci., 18 (1995), 249-957.

[ 6 ] A. Chrzęszczyk, Some existence results in dynamical thermoelasticity, Part 1, Nonlinear case, Arch. Mech., 39 (1987), 605-617.

[ 7 ] A. Chrzęszczyk, Well-posedness of the equations of generalized thermoelasticity, J. Elasticity, 30 (1993), 69-80.

[ 8 ] C.M. Dafermos and W.J. Hrusa, Energy methods for quasilinear hyperbolic initialboundary value problems; Applications to elastodynamics, Arch. Rational Mech. Anal., 87 (1985), 267-292.

[ 9 ] C.M. Dafermos and L. Hsiao, Development of singularities in solutions of the equations of nonlinear thermoelasticity, Quart. Appl. Math., 44 (1986), 463-474.

[10] J. Gawinecki, Global solution to the Cauchy problem in non-linear hyperbolic thermoelasticity, Math. Meth. in the Appl. Sci., 15 (1992), 223-237.

[11] J. Gawinecki, Do Duc Hung and A. Piskorek, The initial-value problem in non-linear hyperelasticity, Bul. Pol. Ac.: Tech., 39 (1991), 17-26.

[12] J. Gawinecki and A. Piskorek, On the initial value problem in geometrically nonlinear elasticity, Bul. Pol. Ac.; Tech., 39 (1991), 7-15.

[13] W.J. Hrusa and S.A. Messaoudi, On formation of singularities in one-dimensional nonlinear thermoelasticity, Arch. Rational Mech. Anal., 111 (1990), 135-151.

[14] S. Jiang and R. Racke, On some quasilinear hyperbolic-parabolic initial boundary value problems, Math. Meth. in the Appl. Sci., 12 (1990), 315-339.

[15] T. Kato, Abstract differential equations and nonlinear mixed problems, Lezioni Fermiane, Pisa, 1985.

[16] S. Klainerman and M. Machedon, Space-time estimates for null forms and the local existence theorem, Comm. Pure Appl. Math., 46 (1993), 1221-1268.

[17] H. Koch, Mixed problems for fully non-linear hyperbolic equations, Math. Z., 214 (1993), $9-42$.

[18] M. Krzyżański and J. Schauder, Quasilineare Differentialgleichungen zweiter Ordnung vom hyperbolischen Typus, Gemische Randwertaufgaben, Studia Math., 6 (1936), 162-189.

[19] J.L. Lions, Quelques mèthodes de resolution des problèmes aux limites non linèaires, Gauthier-Villars, Paris, 1969.

[20] J.L. Lions and E. Magenes, Problèmes aux limites non homogènes et applicationes, Vol 1, Dunod, Paris, 1968. 
[21] A. Majda and E. Thomann, Multi-dimensional shock fronts for second order wave equations, Comm. Partial Differential Equations, 12 (1987), 777-828.

[22] A. Milani and Y. Shibata, On compatible regularizing data for second order hyperbolic initial-boundary value problems, Preprint, 1993.

[23] A. Milani and Y. Shibata, On the strong well-posedness of quasilinear hyperbolic initialboundary value problems, Preprint, 1993.

[24] S.M. Nikol'skij, Approximation of Functions of Several Variables and Imbedding Theorems, Springer Verlag, Berlin, Heidelberg, New York, 1975.

[25] G. Ponce and R. Racke, Global existence of small solutions to the initial value problem for nonlinear thermoelasticity, J. Differential Equations, 87 (1990), 70-83.

[26] R. Racke, Blow-up in nonlinear three-dimensional thermoelasticity, Math. Meth. in the Appl. Sci., 12 (1990), 267-273.

[27] R. Racke, Lectures on Nonlinear Evolution Equation, Initial Value Problems, Friedr. Vieweg \& Sohn, Braunschweig, Wiesbaden, 1992.

[28] Y. Shibata, On a local existence theorem for some quasilinear hyperbolic-parabolic coupled systems with Neumann type boundary condition, Manuscript, 1989.

[29] Y. Shibata, On the Neumann problem for some linear hyperbolic systems of 2nd order with coefficients in Sobolev spaces, Tsukuba J. Math., 13 (1989), 283-352.

[30] Y. Shibata and M. Kikuchi, On the mixed problem for some quasilinear hyperbolic systems with nonlinear boundary condition, J. Differential Equations, 80 (1989), 154-197.

[31] Y. Shibata and Y. Tsutsumi, Local existence of solutions for the initial boundary value problem of fully nonlinear wave equation, Nonlinear Analysis, T.M.A., 11 (1987), 335365.

[32] Y. Shibata and S. Zheng, On some nonlinear hyperbolic systems with damping boundary condition, Nonlinear Analysis, T.M.A., 17 (1991), 233-266.

[33] W. Sickel, Superposition of Functions in Sobolev Spaces of Fractional Order, A survey, Partial Differential Equations, Banach Center Publications, Vol. 27, Part, 2, Warszawa 1992, 481-497.

[34] M.E. Taylor, Pseudodifferential Operators and Nonlinear PDE, Birkhäuser, Boston, 1991.

[35] H. Triebel, Interpolation Theory, Function Spaces, Differential Operators, VEB Deutscher Verlag der Wissenschaften, Berlin, 1978.

[36] H. Triebel, Theory of Function Spaces, Akademische Verl., Geest \& Portig K.-G., Leipzig, 1983.

\author{
INSTITUTE OF MATHEMATICS \\ PEdAgogical UNIVERSITY \\ KONOPNICKIEJ 21 \\ 25-406 KielCe, PolAND
}

